\title{
Partial Applicative Theories and Explicit Substitutions
}

\author{
THOMAS STRAHM, Institut für Informatik und angewandte Mathematik, \\ Universität Bern Länggassstrasse 51, CH-3012 Bern, Switzerland. \\ Email: strahm@iam.unibe.ch
}

\begin{abstract}
Systems based on theories with partial self-application are relevant to the formalization of constructive mathematics and as a logical basis for functional programming languages. In the literature they are either presented in the form of partial combinatory logic or the partial $\lambda$ calculus, and sometimes these two approaches are erroneously considered to be equivalent. In this paper we address some defects of the partial $\lambda$ calculus as a constructive framework for partial functions. In particular, the partial $\lambda$ calculus is not embeddable into partial combinatory logic and it lacks the standard recursion-theoretic model. The main reason is a concept of substitution, which is not consistent with a strongly intensional point of view. We design a weakening of the partial $\lambda$ calculus, which can be embedded into partial combinatory logic. As a consequence, the natural numbers with partial recursive function application are a model of our system. The novel point will be the use of explicit substitutions, which have previously been studied in the literature in connection with the implementation of functional programming languages.
\end{abstract}

Keywords: Explicit mathematics, logic of partial terms, partial $\lambda$ calculus, partial combinatory logic, explicit substitutions.

\section{Introduction}

Partial applicative theories form the basis of various formal systems for constructive mathematics and functional programming. Feferman introduced in [6] and [7] partial applicative theories of operations and classes in order to give a logical account to Bishop's style of constructive mathematics (BCM). More recently, Feferman's systems of explicit mathematics were used to develop a unitary axiomatic framework for representing programs, stating properties of programs, and proving properties of programs. The programs considered are taken from functional programming languages, which are mainly based on the untyped $\lambda$ calculus. Important references for the use of systems of explicit mathematics in the context of functional programming are Feferman $[8,9,10]$, Jäger $[16,17]$ and Marzetta $[22,23]$.

As far as the explicit representation of a theory with partial self-application in the previous literature is concerned, people either took partial combinatory logic or the partial $\lambda$ calculus (without the rule $\xi$ ) as the applicative basis. The former possibility was chosen in $[2,6,7$, $11,16,17,23,28]$, the latter in $[8,9,10,22]$. At first sight, these two approaches seem to be completely equivalent, and sometimes they are treated as such in the literature. But they are only equivalent in the presence of a total logic, since then $\lambda$ calculus (without $\xi$ ) can be embedded into combinatory logic and vice versa. For a detailed discussion of the total case also in the context of reductions, the reader is referred to Hindley [14], and Hindley and Longo [15].

The situation changes drastically if one considers a partial application operation. Then the partial $\lambda$ calculus (without $\xi$ ) is no longer embeddable into partial combinatory logic. This is due to the fact that the coding of $\lambda$ abstraction in the context of partial combinatory logic 
is more complicated than usual. The modified definition of $\lambda$ does not permit one to push a substitution $\theta$ inside an abstraction ( $\lambda x . t)$, a principle, which is valid for the partial $\lambda$ calculus for obvious reasons. For example, the terms $(\lambda x . y)[z z / y]$ and $(\lambda x . z z)$ are not equal in partial combinatory logic. Hence, the stronger concept of substitution in the partial $\lambda$ calculus makes its embedding into partial combinatory logic fail. For the same reason, the standard recursion-theoretic model of partial combinatory logic is no longer a model of the partial $\lambda$ calculus. Recently, Pezzoli [Private communication] even proved that there is no (reasonable) recursion-theoretic interpretation of the partial $\lambda$ calculus at all.

As a consequence, it is not possible to determine proof-theoretical upper bounds of applicative theories based on the partial $\lambda$ calculus by means of the recursion-theoretic model, as can be done for the corresponding systems based on partial combinatory logic. Although the upper bounds of those systems can be determined by formalizing a total term model (cf. Jäger and Strahm [18]), the adequacy of the partial $\lambda$ calculus as a constructive framework for partial functions is seriously put into question. The system simply does not seem to have any reasonable models with a perspicuous constructive meaning that are truly partial. Not only the recursion-theoretic model but also other partial models of partial combinatory logic do not have their counterparts as models of the partial $\lambda$ calculus.

In the following we propose a modification of the partial $\lambda$ calculus, which can be embedded into partial combinatory logic via a natural embedding. As a consequence, this weakened form of the partial $\lambda$ calculus has all the partial models which we have for partial combinatory logic. In particular, it is possible to determine upper bounds of systems of explicit mathematics based on our modified version of the partial $\lambda$ calculus using the recursion-theoretic model.

The novel point of our system will be the use of explicit substitutions. According to this approach substitution is no longer a notion of the metalanguage, but an operation axiomatized in the theory under consideration. If $t, s_{1}, \ldots, s_{n}$ are terms and $\theta$ is the substitution $\left\{s_{1} / x_{1}, \ldots, s_{n} / x_{n}\right\}$ then $t \theta$ is no longer an abbreviation in the metalanguage for the term $t$ with the variables $x_{\imath}$ simultaneously replaced by the terms $s_{i}$, but a purely syntactical object. The evaluation of $\theta$ has to be described by appropriate axioms or rules. So it is possible to provide a very controlled process of substitution. In particular, substitution can be axiomatized in a way that is consistent with the recursion-theoretic model and partial combinatory logic, respectively. Hence, a substitution $\theta$ can no longer be pushed inside an abstraction $(\lambda x . t)$.

The theory of explicit substitutions has been treated in the literature before, but from a different point of view. The main work has been done in the context of implementation of functional programming languages, and application in those systems is always total. The very concern of the present work, however, is to study a partial application operation. A key reference for the previous work on explicit substitution is the paper by Abadi et al. [1]. Further investigations are presented in Curien [4], Curien et al. [5], Hardin and Lévy [13] as well as in Lescanne and Rouyer [20].

Recently, Martin-Löf [21] introduced a calculus of explicit substitutions in connection with his intuitionistic theory of types, which is worked out in Tasistro [26].

Let us briefly sketch the procedure of these investigations. In Section 2 we first introduce partial combinatory logic $\mathrm{CL}_{p}$ and the (usual) partial $\lambda$ calculus $\lambda_{p}$ (without $\xi$ ). In particular, we recapitulate Beeson's logic of partial terms. After having sketched some interesting partial models of $\mathrm{CL}_{p}$, we discuss the substitution problems, which prevent the embedding of $\lambda_{p}$ into $C L_{p}$, and we give Pezzoli's result mentioned above. In Section 3 we give a detailed formulation of the system $\lambda_{p} \sigma$, which is a modification of $\lambda_{p}$ by explicit substitutions. The system incorporates an adaptation of Beeson's logic of partial terms to the framework of ex- 
plicit substitutions, and rules to evaluate substitutions, of course. We further show that $\lambda_{p} \sigma$ is embeddable into $C_{p}$ via a natural embedding and that $\mathrm{CL}_{p}$ is also contained in $\lambda_{p} \sigma$ via the standard embedding. In Section 4, finally, we study the reduction relation on $\lambda_{p} \sigma$ terms corresponding to the system $\lambda_{p} \sigma$. We give a long and tedious proof for the Church Rosser property of this relation.

We finish this introduction by mentioning that recently Stärk (manuscript in preparation) has established a natural relationship between the $\lambda_{p} \sigma$ calculus and the programming language SCHEME.

\section{The systems $\mathrm{CL}_{p}$ and $\lambda_{p}$}

Let us first define partial combinatory logic $\mathrm{CL}_{p}$ and the usual partial $\lambda$ calculus $\lambda_{p}$, without the rule $\xi$. The language of $\mathrm{CL}_{p}$ includes an infinite list of object variables (in the metalanguage: $x, y, z, f, g, h, u, v, w, \ldots$ ), constants $\mathrm{k}$ and $\mathrm{s}$ (partial combinatory algebra), the binary function symbol o (application), the equality symbol $=$, the symbol $\downarrow$ (defined) and the usual propositional connectives and first-order quantifiers. The language of $\lambda_{p}$ contains the same symbols except that $k$ and $s$ are replaced by the abstractor $\lambda$.

The terms of $\mathrm{CL}_{p}$ and $\lambda_{p}$ (in the metalanguage: $r, s, t, \ldots$ ) are given by the following definitions.

DEFINITION 2.1 ( $\mathrm{CL}_{p}$ terms)

1. Every variable is a $\mathrm{CL}_{p}$ term.

2. $k$ and $s$ are $C L_{p}$ terms.

3. If $s$ and $t$ are $\mathrm{CL}_{p}$ terms, then $(s \circ t)$ is a $\mathrm{CL}_{p}$ term.

DEFINITION 2.2 ( $\lambda_{p}$ terms)

1. Every variable is a $\lambda_{p}$ term.

2. If $t$ is a $\lambda_{p}$ term, then $(\lambda x . t)$ is a $\lambda_{p}$ term.

3. If $s$ and $t$ are $\lambda_{p}$ terms, then $(s \circ t)$ is a $\lambda_{p}$ term.

In the following we write $(s t)$ for $(s \circ t)$. Additionally, we adopt the convention of association to the left, i.e. $t_{1} t_{2} t_{3} \ldots t_{n}$ stands for $\left(\ldots\left(\left(t_{1} t_{2}\right) t_{3}\right) \ldots t_{n}\right)$. Finally, we often write $\left(\lambda x_{1} \ldots x_{n} . t\right)$ instead of $\lambda x_{1} \cdot\left(\lambda x_{2} \cdot\left(\ldots\left(\lambda x_{n} \cdot t\right) \ldots\right)\right)$.

The formulas of $\mathrm{CL}_{p}$ (in the metalanguage: $A, B, C, \ldots$ ) are defined in the obvious way.

DEFINITION $2.3\left(\mathrm{CL}_{p}\right.$ formulas)

1. If $s$ and $t$ are $\mathrm{CL}_{p}$ terms, then $(s=t)$ is a $\mathrm{CL}_{p}$ formula.

2. If $t$ is a $\mathrm{CL}_{p}$ term, then $t \downarrow$ is a $\mathrm{CL}_{p}$ formula.

3. If $A$ and $B$ are $\mathrm{CL}_{p}$ formulas, then $\neg A,(A \vee B),(A \wedge B)$ and $(A \rightarrow B)$ are $\mathrm{CL}_{p}$ formulas.

4. If $A$ is a $\mathrm{CL}_{p}$ formula, then $\exists x A$ and $\forall x A$ are $\mathrm{CL}_{p}$ formulas.

The formulas of $\lambda_{p}$ are defined in exactly the same way as the $\mathrm{CL}_{p}$ formulas.

If $t$ is a term of $\mathrm{CL}_{p}$ or $\lambda_{p}$, then fvar $(t)$ denotes the set of free variables of $t$. As usual, $t\left[s_{1} / x_{1}, \ldots, s_{n} / x_{n}\right]$ is the term $t$ in which the free occurrences of $x_{1}, \ldots, x_{n}$ are simultaneously substituted by $s_{1}, \ldots, s_{n}$. If $t$ is a $\lambda_{p}$ term, then in the case of variable collisions bound variables have to be renamed. Analogously, $f v a r(A)$ and $A\left[s_{1} / x_{1}, \ldots, s_{n} / x_{n}\right]$ are defined.

DEFINITION 2.4

1. $(A \leftrightarrow B):=((A \rightarrow B) \wedge(B \rightarrow A))$. 
58 Partial Applicative Theories and Explicit Substitutions

2. $(t \simeq s):=((t \downarrow \vee s \downarrow) \rightarrow t=s)$.

Together with the axioms stated below it will become clear that $(t \simeq s)$ is equivalent to

$$
(t \downarrow \wedge s \downarrow \wedge t=s) \dot{\vee}(\neg t \downarrow \wedge \neg s \downarrow),
$$

i.e. $\simeq$ is a partial equality relation as it is used, for example, in a recursion-theoretic framework.

In the following we give the axioms and rules of inference of the systems $\mathrm{CL}_{p}$ and $\lambda_{p}$, respectively. The logic of both $\mathrm{CL}_{p}$ and $\lambda_{p}$ is the classical logic of partial terms due to Beeson $[3,2]$. It corresponds to $E^{+}$logic as it is discussed in Troelstra and van Dalen [27]. All our results also hold if intuitionistic logic is chosen as a basis of $\mathrm{CL}_{p}$ and $\lambda_{p}$, respectively.

\section{DEFINITION 2.5}

The system $\mathrm{CL}_{p}$ is formulated in the language of $\mathrm{CL}_{p}$, and contains the following list of axioms and rules of inference.

\section{A. Propositional Logic}

(1) Some complete axiom schemes of classical propositional logic

B. Quantifier axioms

(2) $\forall x A \wedge t \downarrow \rightarrow A[t / x]$

(3) $A[t / x] \wedge t \downarrow \rightarrow \exists x A$

C. Equality axioms

(4) $x=x \quad t=s \rightarrow s=t \quad t=s \wedge s=r \rightarrow t=r$

(5) $t_{1}=s_{1} \wedge t_{2}=s_{2} \rightarrow t_{1} t_{2} \simeq s_{1} s_{2}$

D. Strictness axioms

(6) $x \downarrow$

(7) $t_{1}=t_{2} \rightarrow t_{1} \downarrow \wedge t_{2} \downarrow \quad t_{1} t_{2} \downarrow \rightarrow t_{1} \downarrow \wedge t_{2} \downarrow$

(8) $k \downarrow$ s $\downarrow$ sxy $\downarrow$

E. Partial combinatory algebra

(9) $\mathrm{k} x y \simeq x$

(10) $\mathrm{s} x y z \simeq x z(y z)$

\section{F. Rules of inference}

(11) $\frac{A \quad A \rightarrow B}{B}$

(12) $\frac{A \rightarrow B}{\exists x A \rightarrow B} \quad \frac{A \rightarrow B}{A \rightarrow \forall x B}$

In the inference rules (12) $x$ does not appear free in the conclusion. 


\section{DEFINITION 2.6}

The system $\lambda_{p}$ is formulated in the language of $\lambda_{p}$ and contains the same axioms and rules of inference as $\mathrm{CL}_{p}$ except that the strictness axioms (8) are replaced by

$$
(\lambda x . t) \downarrow
$$

and the axioms of a partial combinatory algebra are replaced by the $\beta$ axiom

$$
\text { (B) } \quad(\lambda x . t) y \simeq t[y / x] .
$$
$(\xi)$,

It is important to notice that in the system $\lambda_{p}$ we do not have the partial analogue of the rule

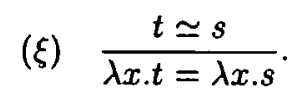

The principle $(\xi)$ induces a weak form of extensionality, which is not consistent with the strongly intensional character of (indices of) partial functions as met, for example, in a recursion-theoretic framework. Formally, this means that it is a priori hopeless to embed $\lambda$ calculus into combinatory logic in the presence of $(\xi)$. Moreover, the absence of $(\xi)$ is in accord with most implementations of $\lambda$ calculus based languages: functions are considered as values, and are only evaluated when arguments are fed in. As already mentioned in the introduction, $\lambda$ calculus without $(\xi)$ in the context of a total application operation is discussed in [14] and [15].

Nevertheless, we will shortly address fully extensional versions of $\mathrm{CL}_{p}$ and $\lambda_{p}$, respectively, i.e. we will consider the strong extensionality axiom (Ext),

$$
\text { (Ext) } \forall x(f x \simeq g x) \rightarrow(f=g) .
$$

Let us briefly sketch some models of $\mathrm{CL}_{p}$. As we are mainly interested in partiality, we will only discuss truly partial models. Of course, there are many models of $\mathrm{CL}_{p}$ where application is a total operation, e.g. each model of the $\lambda$ calculus is a model of $\mathrm{CL}_{p}$. In the following we give two partial models of $\mathrm{CL}_{p}$.

The recursion-theoretic model $P R O$. The universe of the model $P R O$ of partial recursive operations consists of the set of natural numbers $\omega$. Application $\circ$ is interpreted as partial recursive function application, i.e. $x \circ y$ means $\{x\}(y)$ in $P R O$, where $\{x\}$ is a standard enumeration of the partial recursive functions. It is easy to find appropriate interpretations of $k$ and $s$ such that the axioms of a partial combinatory algebra are satisfied. $P R O$ provides a natural example of a domain where objects may be programs as well as inputs to programs. The model underlines the constructive and operational character of applicative theories.

The normal term model $C N T$. This model is based on standard notions of term reduction for combinatory logic, i.e. $k t_{1} t_{2}$ reduces to $t_{1}$ and $s t_{1} t_{2} t_{3}$ reduces to $t_{1} t_{3}\left(t_{2} t_{3}\right)$. The universe of $C N T$ consists of all closed $\mathrm{CL}_{p}$ terms in normal form, $\mathrm{k}$ and $\mathrm{s}$ are interpreted by themselves, and $t_{1} \circ t_{2}$ means InFirst $\left(t_{1} t_{2}\right)$. InFirst $\left(t_{1} t_{2}\right)$ denotes the uniquely determined normal term $s$ provided that $t_{1} t_{2}$ can be reduced to $s$ according to the leftmost minimal strategy, $\operatorname{InFirst}\left(t_{1} t_{2}\right)$ is undefined otherwise. Using the leftmost minimal strategy, at each stage of a reduction sequence the leftmost minimal redex is contracted, where a redex is called minimal, if it does not contain any other redexes. It is necessary to use the leftmost minimal strategy in order to be consistent with the strictness axioms of $\mathrm{CL}_{p}$. The model $C N T$ provides us with another interesting operational semantics of $\mathrm{CL}_{p}$. For a detailed description of $C N T$ the reader is referred to Beeson [2], p. 119. 
As we will see below, the models just described cannot be made into models of the system $\lambda_{p}$ in a reasonable way. This is due to a stronger concept of substitution, which is inherent in the partial $\lambda$ calculus $\lambda_{p}$.

Our next aim is to code $\lambda$ abstraction in $\mathrm{CL}_{p}$ : We have to be careful in defining it in the context of the logic of partial terms, because we want $\lambda x . t$ to be defined for each term $t$. As we will see below, this modified $\lambda$ abstraction will have very unpleasant properties as far as substitution is concerned.

DEFINITION $2.7\left(\lambda^{*} x . t\right)$

For each term $t$ of $\mathrm{CL}_{p}$ a term $\lambda^{*} x . t$ is defined by induction on the complexity of $t$.

1. If $t$ is the variable $x$, then $\lambda^{*} x . t:=$ skk.

2. If $t$ is a variable different from $x$ or a constant, then $\lambda^{*} x . t:=\mathrm{k} t$.

3. If $t$ is the term $\left(t_{1} t_{2}\right)$, then $\lambda^{*} x . t:=\mathrm{s}\left(\lambda^{*} x . t_{1}\right)\left(\lambda^{*} x . t_{2}\right)$.

LEMMA 2.8

We have for all $\mathrm{CL}_{p}$ terms $t$ and $s$ :

1. $f \operatorname{var}\left(\lambda^{*} x . t\right)=f \operatorname{var}(t) \backslash\{x\}$.

2. $C L_{p} \vdash \lambda^{*} x . t \downarrow$.

3. $\mathrm{CL}_{p} \vdash\left(\lambda^{*} x \cdot t\right) x \simeq t$.

4. $\mathrm{CL}_{p} \vdash s \downarrow \rightarrow\left(\lambda^{*} x . t\right) s \simeq t[s / x]$.

PROOF. (1)-(3) are proved by induction on the complexity of $t$. (4) is a direct consequence of (3).

In the context of a total logic, one normally defines $\lambda^{*} x . t:=\mathrm{k} t$, if $x \notin f v a r(t)$. So we have, for example, $\lambda^{*} x .(y z)=k(y z)$. The example shows that $\lambda^{*} x . t \downarrow$ does not hold for the usual definition of $\lambda$ abstraction.

As already mentioned, the $\lambda$ abstraction of Definition 2.7 behaves very badly as far as substitution in $\lambda$ expressions is concerned. For a usual $\lambda$ abstraction we have

$$
(\lambda x . t)[s / y]=\lambda x . t[s / y]
$$

provided that $x \neq y$ and $x \notin f v a r(s)$. This property (which we only need for $s \downarrow$ in a partial setting) fails for the $\lambda^{*}$ defined above: we have, for example, $\left(\lambda^{*} x . y\right)[z z / y]=k(z z)$, but $\lambda^{*} x . z z=\mathrm{s}(\mathrm{k} z)(\mathrm{k} z)$.

The fact that the substitution property (*) does not hold for the $\lambda$ abstraction of Definition 2.7 is not just a technical inconvenience, but has rather strong consequences for the system $\lambda_{p}$ as a constructive framework for partial functions. Since $(*)$ trivially holds in $\lambda_{p}$, the standard embedding of $\lambda_{p}$ into $C L_{p}$ fails. As a consequence, the $C L_{p}$ models $P R O$ and $C N T$ described above do not translate into models of $\lambda_{p}$.

An illustrative consequence of the stronger substitution concept of $\lambda_{p}$ is a very weak form of $(\xi)$, which is derivable in $\lambda_{p}$. Let $s, t$ be $\lambda_{p}$ terms, and $x$ be a variable with $x \notin f v a r(s) \cup$ fvar $(t)$. Then it is easy to see that the principle

$$
s=t \rightarrow \lambda x . s=\lambda x . t
$$

is derivable in $\lambda_{p}$ only from $(\beta)$ and the fact that equality respects application. For $(s=t)$ implies $(\lambda y . \lambda x . y) s \simeq(\lambda y \cdot \lambda x . y) t$, and hence we can conclude by the $\beta$ axiom

$$
\lambda x . s=(\lambda y \cdot \lambda x . y) s=(\lambda y \cdot \lambda x \cdot y) t=\lambda x . t .
$$


Note that in the argument above, we pushed the substitutions $[s / y]$ and $[t / y]$ inside the abstraction $(\lambda x . y)$. The principle (**) can be considered as an extremely weak form of extensionality, and therefore, it has to be rejected from a strongly intensional point of view.

We have seen above that the $\lambda$ abstraction of Definition 2.7 does not yield a recursiontheoretic interpretation of $\lambda_{p}$. The question arises whether it is possible to find another encoding of $\lambda$ in the model $P R O$ satisfying the substitution property (*). It is easily checked that all $\lambda$ encodings known from the literature (cf. Kleene [19], p. 344) do not validate (*), and all attempts to define another such $\lambda$ failed.

Very recently, Pezzoli (private communication) found an elegant formal argument showing that the existence of a recursion-theoretic interpretation of $\lambda_{p}$ which has a partial recursive term evaluation function contradicts the undecidability of the halting problem.

THEOREM 2.9 (Pezzoli)

It is not possible to make the partial recursive functions model $P R O$ of $\mathrm{CL}_{p}$ into a model of the partial $\lambda$ calculus $\lambda_{p}$ in such a way that the term evaluation function $f(t, \rho) \simeq\|t\|_{\rho}$, for $t$ a $\lambda_{p}$ term and $\rho$ an assignment of variables, is partial recursive.

PROOF. Let us assume that we can make the partial recursive functions into a model of $\lambda_{p}$ such that $\|t\|_{\rho}$ is partial recursive. By (4) of Lemma 2.8 and the substitution property (*) we know that $\lambda_{p}$ proves

$$
(z z) \downarrow \rightarrow(\lambda x y \cdot x)(z z)=\lambda y \cdot z z
$$

which immediately yields

$$
(z z) \downarrow \rightarrow \lambda w \cdot((\lambda x y \cdot x)(z z))=\lambda w y \cdot z z
$$

by the principle (**) mentioned above. Now consider $\|\lambda w \cdot((\lambda x y \cdot x)(z z))\|_{\rho}=a_{\rho}$ and $\|\lambda w y . z z\|_{\rho}=b_{\rho}$, which are defined for every $\rho$ since $\lambda$ abstraction is always defined. By $(* * *)$ we have $a_{\rho}=b_{\rho}$ whenever $\{\rho(z)\}(\rho(z)) \downarrow ;$ if $\{\rho(z)\}(\rho(z)) \uparrow$, then $a_{\rho}$ must be an index of the always undefined function and $b_{\rho}$ an index for the constant function $n \mapsto\|\lambda y . z z\|_{\rho}$, so in this case $a_{\rho} \neq b_{\rho}$. However, by hypothesis, we can compute $a_{\rho}$ and $b_{\rho}$, and therefore, we can decide whether $\{n\}(n) \downarrow$. This is not possible.

The following corollary is immediate from the fact that $P R O$ is a model of $\mathrm{CL}_{p}$.

\section{COROLLARY 2.10}

There is no recursive encoding of $\lambda$ in $\mathrm{CL}_{p}$ validating (*).

It should be stressed that the problems described above completely disappear in the presence of the extensionality axiom (Ext). In particular, (*) holds in $\mathrm{CL}_{p}+(\mathrm{Ext})$.

LEMMA 2.11

We have for all $\mathrm{CL}_{p}$ terms $t, s$ and all variables $x, y$ such that $x \neq y$ and $x \notin f v a r(s)$ :

$$
\mathrm{CL}_{p}+(\mathrm{Ext}) \vdash s \downarrow \rightarrow\left(\lambda^{*} x \cdot t\right)[s / y]=\lambda^{*} x \cdot t[s / y]
$$

Proof. Assume $s \downarrow$. By Lemma 2.8 we have $\left(\lambda^{*} x . t\right) x \simeq t$ and $\left(\lambda^{*} x . t[s / y]\right) x \simeq t[s / y]$. Since $s \downarrow$ and $x \neq y$ we can conclude that

$$
\left(\lambda^{*} x \cdot t\right)[s / y] x \simeq t[s / y] \simeq\left(\lambda^{*} x \cdot t[s / y]\right) x,
$$

which by (Ext) and $s \downarrow$ (i.e. $\{\downarrow s\})$ immediately implies the claim of the lemma. 


\section{Partial Applicative Theories and Explicit Substitutions}

As an immediate consequence of this lemma we obtain the following theorem, which is also stated in Moggi [24].

\section{THEOREM 2.12}

The systems $\mathrm{CL}_{p}+(\mathrm{Ext})$ and $\lambda_{p}+($ Ext) are equivalent with respect to the standard embeddings.

\section{The system $\lambda_{p} \sigma$}

In the following, we introduce the system $\lambda_{p} \sigma$, which is a modification of the system $\lambda_{p}$ by explicit substitutions. The language of $\lambda_{p} \sigma$ is an extension of the language of $\lambda_{p}$ by the set brackets $\{$,$\} , the slash / and commas. The new symbols will be used in order to form finite$ sets of variable bindings, i.e. substitutions.

The terms (in the metalanguage: $r, s, t, \ldots$ ) and substitutions (in the metalanguage: $\theta, \sigma, \tau$, $\ldots$ ) of $\lambda_{p} \sigma$ are given by a simultaneous inductive definition.

DEFINITION 3.1 ( $\lambda_{p} \sigma$ terms and $\lambda_{p} \sigma$ substitutions)

1. Every variable is a $\lambda_{p} \sigma$ term.

2. If $t$ is a $\lambda_{p} \sigma$ term, then $(\lambda x . t)$ is a $\lambda_{p} \sigma$ term.

3. If $s$ and $t$ are $\lambda_{p} \sigma$ terms, then $(s \circ t)$ is a $\lambda_{p} \sigma$ term.

4. If $t$ is a $\lambda_{p} \sigma$ term and if $\theta$ is a $\lambda_{p} \sigma$ substitution, then $(t \theta)$ is a $\lambda_{p} \sigma$ term.

5. If $t_{1}, \ldots, t_{n}$ are $\lambda_{p} \sigma$ terms and if $x_{1}, \ldots, x_{n}$ are variables with $x_{i} \neq x_{j}$ for $1 \leq i<j \leq$ $n$, then $\left\{t_{1} / x_{1}, \ldots, t_{n} / x_{n}\right\}$ is a $\lambda_{p} \sigma$ substitution.

In the following we will often write $t \theta \sigma$ instead of $(t \theta) \sigma$.

The formulas of $\lambda_{p} \sigma$ are defined in exactly the same way as the formulas of $\lambda_{p}$, e.g. we have an atomic formula $t \downarrow$ for each $\lambda_{p} \sigma$ term $t$. In the following we often speak of terms, substitutions, and formulas instead of $\lambda_{p} \sigma$ terms, $\lambda_{p} \sigma$ substitutions, and $\lambda_{p} \sigma$ formulas.

We again want to stress the difference between $t[s / x]$ and $t\{s / x\}$. In the first expression substitution is an abbreviation in the metalanguage for the term $t$ with all free occurrences of $x$ replaced by $s, t\{s / x\}$, however, is a purely syntactical object where the substitution $\{s / x\}$ can only be evaluated by means of appropriate axioms to be described below.

We will often use the following abbreviations.

\section{DEFINITION 3.2}

Let $\theta=\left\{t_{1} / x_{1}, \ldots, t_{n} / x_{n}\right\}$ and $\theta^{\prime}=\left\{s_{1} / x_{1}, \ldots, s_{n} / x_{n}\right\}$ be substitutions and let $r$ be an arbitrary term. Then we define:

1. $\operatorname{dom} \theta:=\left\{x_{1}, \ldots, x_{n}\right\}$.

2. $\theta \downarrow:=t_{1} \downarrow \wedge \cdots \wedge t_{n} \downarrow$.

3. $\theta \simeq \theta^{\prime}:=t_{1} \simeq s_{1} \wedge \cdots \wedge t_{n} \simeq s_{n}$.

4. $t / x \cdot \theta:=\{t / x\} \cup \theta^{-x}$, where $\theta^{-x}$ is $\theta$ with a possible binding for $x$ deleted, i.e. $t / x \cdot \theta$ denotes the update of $\theta$ by $\{t / x\}$.

5. $\varepsilon:=\{\}$ (the empty substitution).

The set of free variables fvar $(t)$ of a $\lambda_{p} \sigma$ term $t$ is computed in the obvious way. For reasons of completeness we give the exact inductive definition below.

DEFINITION 3.3 (fvar $(t)$ )

1. If $t$ is the variable $x$, then $f \operatorname{var}(t):=\{x\}$. 
2. If $t$ is the term $(\lambda x . s)$, then fvar $(t):=f v a r(s) \backslash\{x\}$.

3. If $t$ is the term $\left(t_{1} t_{2}\right)$, then fvar $(t):=$ fvar $\left(t_{1}\right) \cup$ fvar $\left(t_{2}\right)$.

4. If $t$ is the term $(s \theta)$ and $\theta=\left\{s_{1} / x_{1}, \ldots, s_{n} / x_{n}\right\}$, then

$$
f \operatorname{var}(t):=(\operatorname{fvar}(s) \backslash \operatorname{dom} \theta) \cup \bigcup_{i \in I} \operatorname{fvar}\left(s_{i}\right),
$$

where $I=\left\{i: 1 \leq i \leq n\right.$ and $\left.x_{i} \in f \operatorname{fvar}(s)\right\}$.

Once fvar $(t)$ is defined, we have $f v a r(A)$ as the set of free variables of a $\lambda_{p} \sigma$ formula $A$ in the usual way. We will sometimes use the notation $\theta\lceil t$ for the substitution $\theta$ with all variable bindings $s / y$ deleted for $y \notin f v a r(t)$.

In the sequel we define a $\lambda_{p} \sigma$ formula $A \theta$ for each $\lambda_{p} \sigma$ formula $A$ and each $\lambda_{p} \sigma$ substitution $\theta$. The definition is by induction on the complexity of $A$.

\section{DEFINITION $3.4(A \theta)$}

1. If $A$ is the formula $(s=t)$, then $A \theta$ is the formula $(s \theta=t \theta)$.

2. If $A$ is the formula $t \downarrow$, then $A \theta$ is the formula $t \theta \downarrow$.

3. If $A$ is the formula $\neg B,(B \vee C),(B \wedge C)$ or $(B \rightarrow C)$, then $A \theta$ is the formula $\neg(B \theta)$, $(B \theta \vee C \theta),(B \theta \wedge C \theta)$ or $(B \theta \rightarrow C \theta)$, respectively.

4. If $A$ is the formula $\exists x B$ or $\forall x B$, then $A \theta$ is the formula $\exists y(B[y / x] \theta)$ or $\forall y(B[y / x] \theta)$ respectively, where $y$ is a 'fresh' variable. ${ }^{1}$

The composition of two substitutions $\theta$ and $\sigma$ is defined in the usual way.

\section{DEFINITION 3.5}

Let $\theta=\left\{t_{1} / x_{1}, \ldots, t_{n} / x_{n}\right\}$ and $\sigma=\left\{s_{1} / y_{1}, \ldots, s_{m} / y_{m}\right\}$ be substitutions. The substitution $\theta \sigma$ is obtained by deleting all variable bindings of the form $s_{i} / y_{i}$ in the set

$$
\left\{t_{1} \sigma / x_{1}, \ldots, t_{n} \sigma / x_{n}, s_{1} / y_{1}, \ldots, s_{m} / y_{m}\right\}
$$

such that $y_{i}=x_{j}$ for a $1 \leq j \leq n$.

Now we are ready to give the exact formulation of the system $\lambda_{p} \sigma$. The logic of $\lambda_{p} \sigma$ is an adaptation of Beeson's logic of partial terms to the framework of explicit substitutions. The novel point of this axiom system compared to the system $\lambda_{p}$ are the substitution axioms $(E)$, which incorporate rules to evaluate substitutions step by step. Furthermore, an extended form of the $\beta$ axiom in the context of explicit substitutions is given.

\section{DEFINITION 3.6}

The system $\lambda_{p} \sigma$ is formulated in the language of $\lambda_{p} \sigma$ and contains the following list of axioms and rules of inference.

\section{A. Propositional logic}

(1) Some complete axiom schemes of classical propositional logic

B. Quantifier axioms

(2) $\forall \vec{x} A \wedge \theta \downarrow \rightarrow A \theta$

\footnotetext{
${ }^{1} B[y / x]$ is the formula $B$, where each free occurrence of $x$ is replaced by $y$ in the usual sense. The exact definition of $B[y / x]$ is straightforward, but tedious.
} 
(3) $A \theta \wedge \theta \downarrow \rightarrow \exists \vec{x} A \quad\left(\vec{x}=x_{1}, \ldots, x_{n} ; \operatorname{dom} \theta=\{\vec{x}\}\right)$

C. Equality axioms

(4) $x=x \quad t=s \rightarrow s=t \quad t=s \wedge s=r \rightarrow t=r$

(5) $t_{1}=s_{1} \wedge t_{2}=s_{2} \rightarrow t_{1} t_{2} \simeq s_{1} s_{2}$

(6) $\theta \simeq \theta^{\prime} \rightarrow t \theta \simeq t \theta^{\prime}$

D. Strictness axioms

(7) $x \downarrow$

(8) $t_{1}=t_{2} \rightarrow t_{1} \downarrow \wedge t_{2} \downarrow \quad t_{1} t_{2} \downarrow \rightarrow t_{1} \downarrow \wedge t_{2} \downarrow$

(9) $(\lambda x . t) \downarrow$

E. Substitution axioms

(10) $x \theta \simeq t \quad(t / x \in \theta)$

(11) $(t s) \theta \simeq(t \theta)(s \theta)$

(12) $(t \theta) \sigma \simeq t(\theta \sigma)$

(13) $t(s / x \cdot \theta) \simeq t \theta \quad(x \notin f \operatorname{fvar}(t) \cup \operatorname{dom} \theta)$

(14) $t \varepsilon \simeq t$

F. $\beta$ axiom

(15) $(\lambda x . t) \theta y \simeq t(y / x \cdot \theta)$

G. Rules of inference

(16) $\frac{A \quad A \rightarrow B}{B}$

(17) $\frac{A \rightarrow B}{\exists x A \rightarrow B} \quad \frac{A \rightarrow B}{A \rightarrow \forall x B}$

In the inference rules (17) $x$ does not appear free in the conclusion.

It should be observed that among the substitution axioms $(E)$ we do not have an axiom which allows us to push a substitution $\theta$ inside an abstraction $(\lambda x . t)$. This is exactly what we want to prevent. Terms of the form $(\lambda x . t) \theta$ can only be resolved if applied to another object, say $y$. This is reflected in the extended $\beta$ axiom (15), where an interleaving substitution $\theta$ is allowed. If $\theta$ is the empty substitution $\varepsilon$ then we have the usual $\beta$ axiom.

Weak $\lambda$ calculi (i.e. $\lambda$ calculi without $(\xi)$ or substitution under $\lambda$ ) with explicit substitutions have been considered in the literature before. These include Curien's $\lambda \rho$ calculus [4], the conditional weak theory $\lambda \sigma_{c w}$ in Curien et al. [5] as well as the weak theory $\lambda \sigma_{w}$ of [5]. The main difference (among other minor differences) between $\lambda \rho, \lambda \sigma_{c w}, \lambda \sigma_{w}$ and $\lambda_{p} \sigma$ lies in the fact that application in the former three calculi is always total, whereas the main concern of the $\lambda_{p} \sigma$ calculus is to model a partial application operation. As we argued in the previous section, such a partial $\lambda$ calculus must not allow $(\xi)$ and substitution under $\lambda$ in order to be consistent with the intended recursion-theoretic interpretation.

LEMMA 3.7

We have for all terms $t, s$ and all substitutions $\theta$ : 
1. $\lambda_{p} \sigma \vdash s \downarrow \rightarrow(\lambda x . t) s \simeq t\{s / x\}$.

2. $\lambda_{p} \sigma \vdash s \downarrow \rightarrow(\lambda x . t) \theta s \simeq t(s / x \cdot \theta)$.

3. $\lambda_{p} \sigma \vdash t \theta \simeq t \quad(\operatorname{dom} \theta \cap f v a r(t)=\emptyset)$.

Proof. By easy reasoning in $\lambda_{p} \sigma$.

In the following we give an embedding $(\cdot)^{C L}$ of the system $\lambda_{p} \sigma$ into partial combinatory logic $\mathrm{CL}_{p}$. This embedding is made possible by a careful concept of substitution in the system $\lambda_{p} \sigma$ corresponding to substitution in $\mathrm{CL}_{p}$. As an immediate consequence of this interpretation we have that the recursion-theoretic model $P R O$ and the normal term model $C N T$ are models of $\lambda_{p} \sigma$. This makes $\lambda_{p} \sigma$ into a system with a reasonable computational and constructive meaning.

Let us first define a $\mathrm{CL}_{p}$ term $t^{C L}$ for each $\lambda_{p} \sigma$ term $t$. The definition is by induction on the complexity of $t$.

\section{DEFINITION $3.8\left(t^{C L}\right)$}

1. If $t$ is a variable, then $t^{C L}:=t$.

2. If $t$ is the term $(\lambda x . s)$, then $t^{C L}:=\left(\lambda^{*} x . s^{C L}\right)$.

3. If $t$ is the term $\left(t_{1} t_{2}\right)$, then $t^{C L}:=\left(t_{1}^{C L} t_{2}^{C L}\right)$.

4. If $t$ is the term $(s \theta)$ where $\theta=\left\{s_{1} / x_{1}, \ldots, s_{n} / x_{n}\right\}$, then $t^{C L}$ is the term ${ }_{s}^{C L}\left[s_{1}^{C L} / x_{1}, \ldots, s_{n}^{C L} / x_{n}\right]$.

Once $(\cdot)^{C L}$ is defined for terms of $\lambda_{p} \sigma$, the translation for formulas is uniquely determined by the requirement that it commutes with $=, \downarrow$, the logical connectives, and the quantifiers. Hence, we have a $C L_{p}$ formula $A^{C L}$ for each $\lambda_{p} \sigma$ formula $A$. From the definition of $(\cdot)^{C L}$ it is immediate that fvar $\left(t^{C L}\right)=f \operatorname{fvar}(t), f \operatorname{viar}\left(A^{C L}\right)=\operatorname{fvar}(A)$, and $A\left\{t_{1} / x_{1}, \ldots, t_{n} / x_{n}\right\}^{C L}=$ $A^{C L}\left[t_{1}^{C L} / x_{1}, \ldots, t_{n}^{C L} / x_{n}\right]$.

We are ready to state the embedding theorem.

\section{THEOREM 3.9}

We have for all $\lambda_{p} \sigma$ formulas $A$ :

$$
\lambda_{p} \sigma \vdash A \quad \Longrightarrow \quad L_{p} \vdash A^{C L}{ }^{2}
$$

Proof. By induction on the length of a proof of $A$ in $\lambda_{p} \sigma$. The propositional axioms do not cause any problems, of course. Also the quantifier axioms are easily handled using the properties of $(\cdot)^{C L}$ mentioned above. The equality axioms are trivial, and in order to establish the translation of $(\lambda x . t) \downarrow$ one makes immediate use of Lemma 2.8 . The substitution axioms are easily verified, too. The extended $\beta$ axiom (15) is treated in exactly the same way as in the proof of Lemma 2.11. Finally, it is trivial to check the inference rules.

In the sequel we show that $\lambda_{p} \sigma$ also includes $\mathrm{CL}_{p}$ by giving an embedding $(\cdot)^{\lambda}$ from $\mathrm{CL}_{p}$ into $\lambda_{p} \sigma$. We first give the translation $(\cdot)^{\lambda}$ for terms of $\mathrm{CL}_{p}$.

DEFINITION $3.10\left(t^{\lambda}\right)$

1. If $t$ is a variable, then $t^{\lambda}:=t$.

2. If $t$ is the constant $k$, then $t^{\lambda}:=\lambda u v . u$.

\footnotetext{
${ }^{2}$ Notice that the converse of this theorem does not hold. To see this, take, for example, for $A$ the formula $x=$ $y \rightarrow \lambda x . x=\lambda z \cdot y$.
} 
66 Partial Applicative Theories and Explicit Substitutions

3. If $t$ is the constant $\mathrm{s}$, then $t^{\lambda}:=\lambda u v w . u w(v w)$.

4. If $t$ is the term $\left(t_{1} t_{2}\right)$, then $t^{\lambda}:=\left(t_{1}^{\lambda} t_{2}^{\lambda}\right)$.

For formulas, $(\cdot)^{\lambda}$ is given in the obvious way, i.e. $(\cdot)^{\lambda}$ commutes with $=, \downarrow$, the logical connectives, and the quantifiers. Again it is obvious that $f \operatorname{ar}\left(t^{\lambda}\right)=\operatorname{fvar}(t)$ and $\operatorname{fvar}\left(A^{\lambda}\right)=$ fvar $(A)$.

\section{LEMMA 3.11}

We have for all $\mathrm{CL}_{p}$ terms $t$ and $s$ :

$$
\lambda_{p} \sigma \vdash t[s / x]^{\lambda} \simeq t^{\lambda}\left\{s^{\lambda} / x\right\}
$$

PROOF. By straightforward induction on the complexity of $t$. Essential use is made of the substitution axioms, in particular axiom (13).

Corollary 3.12

We have for all $\mathrm{CL}_{p}$ formulas $A$, and for all $\mathrm{CL}_{p}$ terms $t$ :

$$
\lambda_{p} \sigma \vdash A[t / x]^{\lambda} \leftrightarrow A^{\lambda}\left\{t^{\lambda} / x\right\}
$$

Proof. By an easy induction on the complexity of $A$ using the above lemma.

\section{LEMMA 3.13}

1. $\lambda_{p} \sigma \vdash(\mathrm{k} x y \simeq x)^{\lambda}$.

2. $\lambda_{p} \sigma \vdash(s x y z \simeq x z(y z))^{\lambda}$.

3. $\lambda_{p} \sigma \vdash(s x y \downarrow)^{\lambda}$.

PROOF. We only prove (1). The proof of (2) and (3) is similar. As we will see, essential use is made of the extended $\beta$ axiom (15). First of all we have

$$
(\lambda u v . u) x \simeq(\lambda v \cdot u)\{x / u\},
$$

which by axiom (15) immediately implies

$$
(\lambda u v . u) x y \simeq(\lambda v . u)\{x / u\} y \simeq u\{x / u, y / v\} .
$$

Furthermore, we have

$$
u\{x / u, y / v\} \simeq x
$$

This finishes the proof of our claim.

LEMMA 3.14

We have for all $\mathrm{CL}_{p}$ formulas $A$ :

$$
\mathrm{CL}_{p} \vdash A \Longrightarrow \lambda_{p} \sigma \vdash A^{\lambda}
$$

Proof. By induction on the length of a proof of $A$ in $\mathrm{CL}_{p}$. Again the propositional axioms are trivial. The translation of the quantifier axioms is provable in $\lambda_{p} \sigma$ by Corollary 3.12. The same corollary also helps in establishing the equality axiom (6). The strictness axioms (9) and the axioms for a partial combinatory algebra were already treated in Lemma 3.13. The inference rules of $\mathrm{CL}_{p}$ readily translate into inference rules of $\lambda_{p} \sigma$. 
The converse of the above lemma also holds.

LEMMA 3.15

We have for all $\mathrm{CL}_{p}$ formulas $A$ :

$$
\lambda_{p} \sigma \vdash A^{\lambda} \quad \Longrightarrow \quad \mathrm{CL}_{p} \vdash A
$$

Proof. We define a modification $(\cdot)^{\bullet}$ of the translation $(\cdot)^{C L}$ from $\lambda_{p} \sigma$ into $\mathrm{CL}_{p} \cdot(\cdot)^{\bullet}$ is defined in the same way as $(\cdot)^{C L}$, except that it uses the more complicated coding $\lambda^{\bullet}$ of $\lambda$ abstraction instead of $\lambda^{*}$. The term $\lambda^{*} x . t$ is inductively defined as follows.

1. If $t$ is the variable $x$, then $\lambda^{\bullet} x . t:=$ skk.

2. If $t$ is a variable different from $x$ or a constant, then $\lambda^{\bullet} x . t:=\mathrm{kt}$.

3. If $t$ is the term $(s x)$, where $s \in\{y, k, s, s y\}$ and $y \neq x$, then $\lambda^{\bullet} x . t:=s$.

4. If $t$ is the term $\left(t_{1} t_{2}\right)$, and if (3) does not apply, then $\lambda^{\bullet} x . t:=s\left(\lambda^{\bullet} x \cdot t_{1}\right)\left(\lambda^{\bullet} x . t_{2}\right)$.

One easily verifies that $\left(\mathrm{k}^{\lambda}\right)^{\bullet}=\mathrm{k}$ and $\left(\mathrm{s}^{\lambda}\right)^{\bullet}=\mathrm{s}$. As an immediate consequence we have $\left(t^{\lambda}\right)^{\bullet}=t$ and $\left(A^{\lambda}\right)^{\bullet}=A$ for all $\mathrm{CL}_{p}$ terms $t$ and all $\mathrm{CL}_{p}$ formulas $A$, respectively. Furthermore, Lemma 2.8 holds for $\lambda^{\bullet}$, too. In particular, we have $\lambda^{\bullet} x . t \downarrow$ for all $C L_{p}$ terms $t$. We can, therefore, establish Theorem 3.9 for $(\cdot)^{\bullet}$ instead of $(\cdot)^{C L}$. Hence, we have

$$
\lambda_{p} \sigma \vdash A \quad \Longrightarrow \quad \mathrm{CL}_{p} \vdash A^{\bullet}
$$

for all $\lambda_{p} \sigma$ formulas $A$. Now the claim of the lemma immediately follows from the fact that $(\cdot)^{\bullet}$ is the inverse of $(\cdot)^{\lambda}$, as we have mentioned above.

Here is the final embedding theorem.

THEOREM 3.16

We have for all $\mathrm{CL}_{p}$ formulas $A$ :

$$
\mathrm{CL}_{p} \vdash A \Longleftrightarrow \lambda_{p} \sigma \vdash A^{\lambda}
$$

PROOF. Immediate from the previous two lemmas.

\section{Confluent reductions}

Once we have introduced the system $\lambda_{p} \sigma$, it is natural to study the corresponding reduction relation on $\lambda_{p} \sigma$ terms. We now define a binary relation $\triangleright$ on $\lambda_{p} \sigma$ terms, which reflects a directed equality relation for the system $\lambda_{p} \sigma . \triangleright$ is defined inductively as follows.

DEFINITION 4.1

The relation $\triangleright$ between $\lambda_{p} \sigma$ terms is generated by the following clauses (1)-(13).

\section{A. Identity}

(1) $t \triangleright t$

B. $\beta$ reductions

(2) $(\lambda x . t) s \triangleright t\{s / x\}$

(3) $(\lambda x . t) \theta s \triangleright t(s / x \cdot \theta)$ 


\section{Substitution reductions}
(4) $x \theta \triangleright t$
$(t / x \in \theta)$
(5) $(t s) \theta \triangleright(t \theta)(s \theta)$
(6) $(t \theta) \sigma \triangleright t(\theta \sigma)$
(7) $t(s / x \cdot \theta) \triangleright t \theta$
$(x \notin f \operatorname{fvar}(t) \cup \operatorname{dom} \theta)$
(8) $t \varepsilon \triangleright t$

\section{Structural rules}

(9) $t \triangleright s=r(t / x \cdot \theta) \triangleright r(s / x \cdot \theta)$
(10) $t \triangleright s \Longrightarrow t \theta \triangleright s \theta$
(11) $t \triangleright s \Longrightarrow r t \triangleright r s$
(12) $t \triangleright s \Longrightarrow t r \triangleright s r$

\section{E. Transitivity}

$$
t \triangleright s, s \triangleright r \Rightarrow t \triangleright r
$$

Notice that we have to state two clauses for $\beta$ reduction, since (2) is no longer derivable from (3) in the context of reductions. Furthermore, it should be observed again that we do not have the rule $(\xi)$,

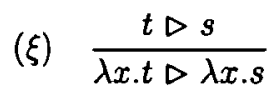

among the structural rules (D).

\section{REMARK 4.2}

We want to stress that the reduction relation $D$ does not take into consideration partiality in $\lambda_{p} \sigma$. Hence, $\triangleright$ rather corresponds to a total version of $\lambda_{p} \sigma$. This is, however, in complete analogy to the system $\mathrm{CL}_{p}$, where truly partial term models are constructed using special reduction strategies of a total reduction relation (cf. the model CNT on p. 59), and partiality is reflected by non-terminating reduction sequences. Summarizing, $\triangleright$ provides a general term reduction framework giving rise to partial and total term models for $\lambda_{p} \sigma$.

In the following $\triangleright_{\sigma}$ denotes the restriction of $\triangleright$ to substitution reductions only, i.e. $t \triangleright_{\sigma} s$ holds if and only if there is a derivation of $t \triangleright s$ according to the above clauses, which does not use (2) and (3). Analogously, $\nabla_{\beta}$ is the restriction of $\triangleright$ to $\beta$ reductions. Finally, we write $t \triangleright^{1} s$ if $t \triangleright s$ is derivable from (2)-(12), i.e. $\triangleright^{1}$ denotes one step reduction. The relations $\nabla_{\sigma}^{1}$ and $D_{\beta}^{1}$ are defined in the same way.

In the sequel we want to show that $\triangleright$ satisfies the Church Rosser property. As usual, this will guarantee that all terminating rewrite sequences yield identical results, i.e. we will have uniqueness of normal forms. All attempts to find a direct proof for the confluence of $\square$ failed. In particular, parallelization does not seem to work in order to show confluence of $\lambda_{p} \sigma$. Instead we will make use of an interpretation technique due to Hardin, which was identified in [12]. This method has subsequently been used several times in order to show confluence for systems of explicit substitutions.

A first step towards the proof of the Church Rosser property for $\triangleright$ is to show that $\nabla_{\sigma}$ is Church Rosser. In order to apply an old result by Newman [25], we establish that $\triangleright_{\sigma}^{1}$ is weakly Church Rosser and wellfounded. 


\section{LEMMA 4.3}

$\triangleright_{\sigma}^{1}$ is weakly Church Rosser, i.e. we have for all terms $t, t_{1}, t_{2}$ : If $t \triangleright_{\sigma}^{1} t_{1}$ and $t \triangleright_{\sigma}^{1} t_{2}$, then there is a term $t_{3}$ such that $t_{1} \triangleright_{\sigma} t_{3}$ and $t_{2} \triangleright_{\sigma} t_{3}$.

Proof. One shows by a straightforward, but tedious induction on the length of a derivation of $t \triangleright_{\sigma}^{1} t_{1}$ that for all $t \triangleright_{\sigma}^{1} t_{2}$ there exists a $t_{3}$ such that $t_{1} \triangleright_{\sigma} t_{3}$ and $t_{2} \triangleright_{\sigma} t_{3}$.

In order to prove that $\triangleright_{\sigma}^{1}$ is wellfounded, we define a measure function $\Psi$ from the $\lambda_{p} \sigma$ terms and $\lambda_{p} \sigma$ substitutions to $\omega$.

DEFINITION $4.4(\Psi(t) ; \Psi(\theta))$

1. If $t$ is a variable, then $\Psi(t):=1$.

2. If $t$ is the term $(\lambda x . s)$, then $\Psi(t):=1$.

3. If $t$ is the term $\left(t_{1} t_{2}\right)$, then $\Psi(t):=\Psi\left(t_{1}\right)+\Psi\left(t_{2}\right)+1$.

4. If $t$ is the term $(s \theta)$, then $\Psi(t):=\Psi(s) \cdot(\Psi(\theta)+1)$.

5. If $\theta$ is the substitution $\left\{t_{1} / x_{1}, \ldots, t_{n} / x_{n}\right\}$, then $\Psi(\theta):=\Psi\left(t_{1}\right)+\cdots+\Psi\left(t_{n}\right)+1$.

Notice that $\Psi(t)>0$ for all terms $t$ and $\Psi(\theta)>0$ for all substitutions $\theta$, e.g. we have $\Psi(\varepsilon)=1$. The composition $(\theta \sigma)$ of two substitutions $\theta$ and $\sigma$ is bounded as follows w.r.t. $\Psi$.

\section{LEMMA 4.5}

We have for all substitutions $\theta$ and $\sigma$ :

$$
\Psi(\theta \sigma)<\Psi(\theta) \cdot(\Psi(\sigma)+1) .
$$

Proof. By an easy calculation.

We are ready to prove that $\Psi$ is strictly decreasing on $\nabla_{\sigma}^{\mathbf{1}}$.

LEMMA 4.6

We have for all terms $t$ and $s$ :

$$
t \triangleright_{\sigma}^{1} s \quad \Longrightarrow \quad \Psi(t)>\Psi(s) .
$$

PROOF. By a straightforward induction on the length of a derivation of $t \triangleright_{\sigma}^{1} s$. Let us only discuss the substitution reduction (6), i.e. $t=(r \theta) \sigma$ and $s=r(\theta \sigma)$ for some term $r$. Then we have

$$
\Psi((r \theta) \sigma)=\Psi(r) \cdot(\Psi(\theta)+1) \cdot(\Psi(\sigma)+1)>\Psi(r) \cdot[\Psi(\theta) \cdot(\Psi(\sigma)+1)+1] .
$$

But $\Psi(r) \cdot[\Psi(\theta) \cdot(\Psi(\sigma)+1)+1]>\Psi(r) \cdot[\Psi(\theta \sigma)+1]$ by the previous lemma. The claim is proved, since $\Psi(r) \cdot[\Psi(\theta \sigma)+1]=\Psi(r(\theta \sigma))$.

\section{COROLLARY 4.7}

$\triangleright_{\sigma}^{1}$ is wellfounded.

Together we have established the following theorem.

\section{THEOREM 4.8}

$\nabla_{\sigma}$ is Church Rosser.

PROOF. The theorem is immediate from Lemma 4.3 and Corollary 4.7 and a result by Newman [25] saying that a reduction relation, which is weakly Church Rosser and wellfounded satisfies the full Church Rosser property. 


\section{COROLLARY 4.9}

Every $\lambda_{p} \sigma$ term has a unique substitution normal form.

In the following we denote the substitution normal form of a term $t$ with $\Sigma(t)$. A substitution $\theta=\left\{t_{1} / x_{1}, \ldots, t_{n} / x_{n}\right\}$ is in substitution normal form, if for all $i$ with $1 \leq i \leq n$ the term $t_{i}$ is in substitution normal form. Analogously, $\Sigma(\theta)$ denotes the substitution normal form of a substitution $\theta$.

As a further step towards the Church Rosser property of $\triangleright$ we make use of a relation $\nabla_{\beta_{n}}$, which corresponds to $\beta$ reduction on terms in substitution normal form, i.e. terms satisfying $\Sigma(t)=t$. We define $\triangleright_{\beta_{n}}$ via its "parallel" version $\stackrel{\beta_{n}}{\longrightarrow}$, i.e. $\triangleright_{\beta_{n}}$ will be the transitive closure of $\stackrel{\beta_{n}}{\longmapsto}$. Then the Church Rosser property of $\stackrel{\beta_{n}}{\longmapsto}$ carries over to $D_{\beta_{n}}$ by a well-known diagram chase. For notational convenience we define $\stackrel{\beta_{n}}{\longmapsto}$ on substitutions, too.

DEFINITION 4.10

The relation $\stackrel{\beta_{n}}{\longrightarrow}$ between $\lambda_{p} \sigma$ terms and $\lambda_{p} \sigma$ substitutions in substitution normal form is simultaneously generated by the following clauses $(1)-(6)$ :

A. Terms

(1) $t \stackrel{\beta_{\mathrm{n}}}{\longmapsto} t$

(2) $s \stackrel{\beta_{n}}{\longmapsto} s^{\prime} \quad \Longrightarrow \quad(\lambda x . t) s \stackrel{\beta_{n}}{\longmapsto} \Sigma\left(t\left\{s^{\prime} / x\right\}\right)$

(3) $s \stackrel{\beta_{n}}{\longmapsto} s^{\prime}, \theta \stackrel{\beta_{n}}{\longmapsto} \theta^{\prime} \quad \Longrightarrow \quad(\lambda x . t) \theta s \stackrel{\beta_{n}}{\longmapsto} \Sigma\left(t\left(s^{\prime} / x \cdot \theta^{\prime}\right)\right)$

(4) $\theta \stackrel{\beta_{n}}{\longmapsto} \theta^{\prime} \quad \Longrightarrow \quad(\lambda x . t) \theta \stackrel{\beta_{n}}{\longmapsto}(\lambda x . t) \theta^{\prime}$

(5) $t \stackrel{\beta_{n}}{\longmapsto} t^{\prime}, s \stackrel{\beta_{n}}{\longmapsto} s^{\prime} \quad \Longrightarrow \quad t s \stackrel{\beta_{n}}{\longmapsto} t^{\prime} s^{\prime}$

B. Substitutions

(6) $t_{1} \stackrel{\beta_{n}}{\longmapsto} s_{1}, \ldots, t_{n} \stackrel{\beta_{n}}{\longrightarrow} s_{n} \quad \Longrightarrow \quad\left\{t_{1} / x_{1}, \ldots, t_{n} / x_{n}\right\} \stackrel{\beta_{n}}{\longmapsto}\left\{s_{1} / x_{1}, \ldots, s_{n} / x_{n}\right\}$

DEFINITION 4.11

Let $D_{\beta_{\mathrm{n}}}$ be the transitive closure of $\stackrel{\beta_{\mathrm{n}}}{\longmapsto}$.

LEMMA 4.12

1. If $t \stackrel{\beta_{n}}{\longmapsto} t^{\prime}$ then we have $\Sigma(t \theta) \stackrel{\beta_{n}}{\longrightarrow} \Sigma\left(t^{\prime} \theta^{\prime}\right)$ for all $\theta \stackrel{\beta_{n}}{\longmapsto} \theta^{\prime}$.

2. If $\theta \stackrel{\beta_{n}}{\longrightarrow} \theta^{\prime}$ then we have $\Sigma(\theta \sigma) \stackrel{\beta_{n}}{\longrightarrow} \Sigma\left(\theta^{\prime} \sigma^{\prime}\right)$ for all $\sigma \stackrel{\beta_{n}}{\longrightarrow} \sigma^{\prime}$.

Proof. (1) and (2) are proved simultaneously by induction on the complexity of $t$ and $\theta$, respectively.

The next lemma says that $\stackrel{\beta_{n}}{\longmapsto}$ is Church Rosser on terms and substitutions.

\section{LEMMA 4.13}

1. If $t \stackrel{\beta_{n}}{\longrightarrow} t_{1}$ then for all $t \stackrel{\beta_{n}}{\longrightarrow} t_{2}$ there is a $t_{3}$ such that $t_{1} \stackrel{\beta_{n}}{\longmapsto} t_{3}$ and $t_{2} \stackrel{\beta_{n}}{\longrightarrow} t_{3}$.

2. If $\theta \stackrel{\beta_{n}}{\longmapsto} \theta_{1}$ then for all $\theta \stackrel{\beta_{n}}{\longmapsto} \theta_{2}$ there is a $\theta_{3}$ such that $\theta_{1} \stackrel{\beta_{n}}{\longmapsto} \theta_{3}$ and $\theta_{2} \stackrel{\beta_{n}}{\longmapsto} \theta_{3}$.

PROOF. We prove (1) and (2) simultaneously by induction on $t \stackrel{\beta_{n}}{\longmapsto} t_{1}$ and $\theta \stackrel{\beta_{n}}{\longmapsto} \theta_{1}$, respec-

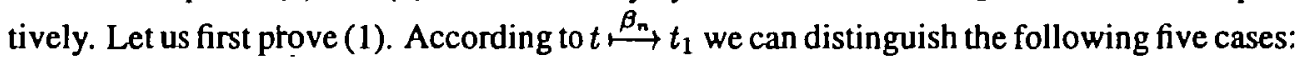

(1) $t \stackrel{\beta_{n}}{\longmapsto} t_{1}$ is $t \stackrel{\beta_{n}}{\longmapsto} t$. Then we can choose $t_{3}:=t_{2}$. 
(2) $t \stackrel{\beta_{n}}{\longmapsto} t_{1}$ is $(\lambda x . r) s \stackrel{\beta_{n}}{\longmapsto} \Sigma\left(r\left\{s^{\prime} / x\right\}\right)$ and is a consequence of $s \stackrel{\beta_{n}}{\longmapsto} s^{\prime}$. According to $t \stackrel{\beta_{n}}{\longmapsto} t_{2}$ we can distinguish the following two subcases:

(2.1) $t \stackrel{\beta_{n}}{\longmapsto} t_{2}$ is $(\lambda x . r) s \stackrel{\beta_{n}}{\longmapsto}(\lambda x . r) s^{\prime \prime}$ and is a consequence of $s \stackrel{\beta_{n}}{\longmapsto} s^{\prime \prime}$. By the induction hypothesis there is a term $s^{\prime \prime \prime}$ with $s^{\prime} \stackrel{\beta_{n}}{\longmapsto} s^{\prime \prime \prime}$ and $s^{\prime \prime} \stackrel{\beta_{n}}{\longmapsto} s^{\prime \prime \prime}$. If we take $t_{3}:=\Sigma\left(r\left\{s^{\prime \prime \prime} / x\right\}\right)$ then obviously $t_{2} \stackrel{\beta_{n}}{\longrightarrow} t_{3}$. By the previous lemma we also have $t_{1} \stackrel{\beta_{n}}{\longmapsto} t_{3}$.

(2.2) $t \stackrel{\beta_{n}}{\longrightarrow} t_{2}$ is $(\lambda x . r) s \stackrel{\beta_{n}}{\longrightarrow} \Sigma\left(r\left\{s^{\prime \prime} / x\right\}\right)$ and is a consequence of $s \stackrel{\beta_{n}}{\longrightarrow} s^{\prime \prime}$. By the induction hypothesis there exists a term $s^{\prime \prime \prime}$ with $s^{\prime} \stackrel{\beta_{n}}{\longmapsto} s^{\prime \prime \prime}$ and $s^{\prime \prime} \stackrel{\beta_{n}}{\longmapsto} s^{\prime \prime \prime}$. Let $t_{3}$ be $\Sigma\left(r\left\{s^{\prime \prime \prime} / x\right\}\right)$. Using the previous lemma one easily verifies $t_{1} \stackrel{\beta_{n}}{\longmapsto} t_{3}$ and $t_{2} \stackrel{\beta_{n}}{\longmapsto} t_{3}$.

(3) $t \stackrel{\beta_{n}}{\longmapsto} t_{1}$ is $(\lambda x . r) \theta s \stackrel{\beta_{n}}{\longmapsto} \Sigma\left(r\left(s^{\prime} / x \cdot \theta^{\prime}\right)\right)$ and is a consequence of $s \stackrel{\beta_{n}}{\longmapsto} s^{\prime}$ and $\theta \stackrel{\beta_{n}}{\longmapsto} \theta^{\prime}$. According to $t \stackrel{\beta_{n}}{\longrightarrow} t_{2}$ we can distinguish the following two subcases:

(3.1) $t \stackrel{\beta_{n}}{\longmapsto} t_{2}$ is $(\lambda x . r) \theta s \stackrel{\beta_{n}}{\longmapsto}(\lambda x . r) \theta^{\prime \prime} s^{\prime \prime}$ and is a consequence of $\theta \stackrel{\beta_{n}}{\longmapsto} \theta^{\prime \prime}$ and $s \stackrel{\beta_{n}}{\longmapsto} s^{\prime \prime}$. By the induction hypothesis there are $s^{\prime \prime \prime}$ and $\theta^{\prime \prime \prime}$ with $s^{\prime} \stackrel{\beta_{n}}{\longmapsto} s^{\prime \prime \prime}, s^{\prime \prime} \stackrel{\beta_{\mathrm{n}}}{\longmapsto} s^{\prime \prime \prime}$ and $\theta^{\prime} \stackrel{\beta_{\mathrm{n}}}{\longmapsto} \theta^{\prime \prime \prime}$, $\theta^{\prime \prime} \stackrel{\beta_{n}}{\longmapsto} \theta^{\prime \prime \prime}$. If we take $t_{3}:=\Sigma\left(r\left(s^{\prime \prime \prime} / x \cdot \theta^{\prime \prime \prime}\right)\right)$ then obviously $t_{2} \stackrel{\beta_{n}}{\longmapsto} t_{3}$. By the fact that $s^{\prime} / x \cdot \theta^{\prime} \stackrel{\beta_{n}}{\longmapsto} s^{\prime \prime \prime} / x \cdot \theta^{\prime \prime \prime}$ and the previous lemma we also have $t_{1} \stackrel{\beta_{m}}{\longmapsto} t_{3}$.

(3.2) $t \stackrel{\beta_{n}}{\longmapsto} t_{2}$ is $(\lambda x . r) \theta s \stackrel{\beta_{n}}{\longmapsto} \Sigma\left(r\left(s^{\prime \prime} / x \cdot \theta^{\prime \prime}\right)\right)$ and is a consequence of $s \stackrel{\beta_{n}}{\longmapsto} s^{\prime \prime}$ and $\theta \stackrel{\beta_{n}}{\longmapsto} \theta^{\prime \prime}$. By the induction hypothesis there are $s^{\prime \prime \prime}$ and $\theta^{\prime \prime \prime}$ with $s^{\prime} \stackrel{\beta_{n}}{\longmapsto} s^{\prime \prime \prime}, s^{\prime \prime} \stackrel{\beta_{n}}{\longmapsto} s^{\prime \prime \prime}$ and $\theta^{\prime} \stackrel{\beta_{n}}{\longmapsto} \theta^{\prime \prime \prime}$, $\theta^{\prime \prime} \stackrel{\beta_{n}}{\longmapsto} \theta^{\prime \prime \prime}$. Let $t_{3}:=\Sigma\left(r\left(s^{\prime \prime \prime} / x \cdot \theta^{\prime \prime \prime}\right)\right)$. Then, by the previous lemma, we have $t_{1} \stackrel{\beta_{n}}{\longmapsto} t_{3}$ and $t_{2} \stackrel{\beta_{n}}{\longmapsto} t_{3}$, since $s^{\prime} / x \cdot \theta^{\prime} \stackrel{\beta_{n}}{\longmapsto} s^{\prime \prime \prime} / x \cdot \theta^{\prime \prime \prime}$ and $s^{\prime \prime} / x \cdot \theta^{\prime \prime} \stackrel{\beta_{n}}{\longmapsto} s^{\prime \prime \prime} / x \cdot \theta^{\prime \prime \prime}$.

(4) $t \stackrel{\beta_{n}}{\longmapsto} t_{1}$ is $(\lambda x . r) \theta \stackrel{\beta_{n}}{\longmapsto}(\lambda x . r) \theta^{\prime}$ and is a consequence of $\theta \stackrel{\beta_{n}}{\longmapsto} \theta^{\prime}$. Then $t \stackrel{\beta_{n}}{\longmapsto} t_{2}$ is $(\lambda x . r) \theta \stackrel{\beta_{n}}{\longmapsto}(\lambda x . r) \theta^{\prime \prime}$ and is a consequence of $\theta \stackrel{\beta_{n}}{\longmapsto} \theta^{\prime \prime}$. By the induction hypothesis there is a $\theta^{\prime \prime \prime}$ with $\theta^{\prime} \stackrel{\beta_{n}}{\longmapsto} \theta^{\prime \prime \prime}$ and $\theta^{\prime \prime} \stackrel{\beta_{n}}{\longmapsto} \theta^{\prime \prime \prime}$. The claim holds for $t_{3}:=(\lambda x . r) \theta^{\prime \prime \prime}$.

(5) $t \stackrel{\beta_{n}}{\longmapsto} t_{1}$ is $r s \stackrel{\beta_{m}}{\longmapsto} r^{\prime} s^{\prime}$ and is a consequence of $r \stackrel{\beta_{n}}{\longmapsto} r^{\prime}$ and $s \stackrel{\beta_{n}}{\longmapsto} s^{\prime}$. According to $t \stackrel{\beta_{n}}{\longmapsto} t_{2}$ we can distinguish the following three subcases:

(5.1) $t \stackrel{\beta_{n}}{\longmapsto} t_{2}$ is $r s \stackrel{\beta_{n}}{\longmapsto} r^{\prime \prime} s^{\prime \prime}$ and is a consequence of $r \stackrel{\beta_{n}}{\longmapsto} r^{\prime \prime}$ and $s \stackrel{\beta_{n}}{\longmapsto} s^{\prime \prime}$. By the induction hypothesis there are terms $r^{\prime \prime \prime}$ and $s^{\prime \prime \prime}$ with $r^{\prime} \stackrel{\beta_{n}}{\longmapsto} r^{\prime \prime \prime}, r^{\prime \prime} \stackrel{\beta_{n}}{\longmapsto} r^{\prime \prime \prime}$ and $s^{\prime} \stackrel{\beta_{n}}{\longmapsto} s^{\prime \prime \prime}, s^{\prime \prime} \stackrel{\beta_{m}}{\longmapsto} s^{\prime \prime \prime}$. The claim immediately follows for $t_{3}:=r^{\prime \prime \prime} s^{\prime \prime \prime}$.

(5.2) $t \stackrel{\beta_{n}}{\longmapsto} t_{2}$ is $\left(\lambda x . r^{\prime \prime}\right) s \stackrel{\beta_{n}}{\longmapsto} \Sigma\left(r^{\prime \prime}\left\{s^{\prime \prime} / x\right\}\right)$ and is a consequence of $s \stackrel{\beta_{n}}{\longmapsto} s^{\prime \prime}$. By the induction hypothesis there is an $s^{\prime \prime \prime}$ with $s^{\prime} \stackrel{\beta_{n}}{\longmapsto} s^{\prime \prime \prime}$ and $s^{\prime \prime} \stackrel{\beta_{n}}{\longmapsto} s^{\prime \prime \prime}$. Furthermore, it is clear that $r=r^{\prime}=\left(\lambda x . r^{\prime \prime}\right)$. If we take $t_{3}:=\Sigma\left(r^{\prime \prime}\left\{s^{\prime \prime \prime} / x\right\}\right)$ then obviously $t_{1} \stackrel{\beta_{n}}{\longmapsto} t_{3}$. By the previous lemma we also have $t_{2} \stackrel{\beta_{n}}{\longmapsto} t_{3}$.

(5.3) $t \stackrel{\beta_{n}}{\longmapsto} t_{2}$ is $\left(\lambda x \cdot r^{\prime \prime}\right) \theta s \stackrel{\beta_{n}}{\longmapsto} \Sigma\left(r^{\prime \prime}\left\{s^{\prime \prime} / x \cdot \theta^{\prime \prime}\right)\right)$ and is a consequence of $s \stackrel{\beta_{n}}{\longmapsto} s^{\prime \prime}$ and $\theta \stackrel{\beta_{n}}{\longmapsto} \theta^{\prime \prime}$. By the induction hypothesis there is an $s^{\prime \prime \prime}$ with $s^{\prime} \stackrel{\beta_{m}}{\longmapsto} s^{\prime \prime \prime}$ and $s^{\prime \prime} \stackrel{\beta_{n}}{\longmapsto} s^{\prime \prime \prime}$. Furthermore, $r \stackrel{\beta_{n}}{\longmapsto} r^{\prime}$ has the form $\left(\lambda x . r^{\prime \prime}\right) \theta \stackrel{\beta_{\mathrm{n}}}{\longmapsto}\left(\lambda x . r^{\prime \prime}\right) \theta^{\prime}$ and is a consequence of $\theta \stackrel{\beta_{\mathfrak{m}}}{\longmapsto} \theta^{\prime}$. By the induction hypothesis there is a $\theta^{\prime \prime \prime}$ with $\theta^{\prime} \stackrel{\beta_{\mathrm{n}}}{\longmapsto} \theta^{\prime \prime \prime}$ and $\theta^{\prime \prime} \stackrel{\beta_{\mathrm{n}}}{\longmapsto} \theta^{\prime \prime \prime}$. If we take $t_{3}:=\Sigma\left(r^{\prime \prime}\left(s^{\prime \prime \prime} / x \cdot \theta^{\prime \prime \prime}\right)\right)$ then obviously $t_{1} \stackrel{\beta_{n}}{\longrightarrow} t_{3}$. By the previous lemma we also have $t_{2} \stackrel{\beta_{n}}{\longrightarrow} t_{3}$. 
This finishes the proof of (1). The proof of (2) is straightforward. Assume $\theta \stackrel{\beta_{n}}{\longrightarrow} \theta_{1}$ and $\theta \stackrel{\beta_{n}}{\longmapsto} \theta_{2}$, where

$$
\begin{aligned}
\theta & =\left\{t_{1} / x_{1}, \ldots, t_{n} / x_{n}\right\} \\
\theta_{1} & =\left\{s_{1} / x_{1}, \ldots, s_{n} / x_{n}\right\} \\
\theta_{2} & =\left\{s_{1}^{\prime} / x_{1}, \ldots, s_{n}^{\prime} / x_{n}\right\}
\end{aligned}
$$

and $t_{i} \stackrel{\beta_{n}}{\longmapsto} s_{i}, t_{i} \stackrel{\beta_{n}}{\longmapsto} s_{i}^{\prime}$ for $1 \leq i \leq n$. By the induction hypothesis there are terms $r_{i}$ with $s_{i} \stackrel{\beta_{n}}{\longrightarrow} r_{i}$ and $s_{i}^{\prime} \stackrel{\beta_{n}}{\longmapsto} r_{i}$ for $1 \leq i \leq n$. If we take $\theta_{3}:=\left\{r_{1} / x_{1}, \ldots, r_{n} / x_{n}\right\}$, then we immediately obtain $\theta_{1} \stackrel{\beta_{\mathrm{n}}}{\longmapsto} \theta_{3}$ and $\theta_{2} \stackrel{\beta_{3}}{\longmapsto} \theta_{3}$.

The proof of the Church Rosser property of $\nabla_{\beta_{n}}$ is complete.

\section{THEOREM 4.14}

$\nabla_{\beta_{n}}$ is Church Rosser.

Proof. By the previous lemma, $\stackrel{\beta_{n}}{\longrightarrow}$ is Church Rosser. By Definition 4.11, $\triangleright_{\beta_{n}}$ is the transitive closure of $\stackrel{\beta_{n}}{\longmapsto}$, which, by a simple diagram chase, implies that $D_{\beta_{n}}$ is confluent, too.

The last step towards the Church Rosser property of $\square$ is to show that

$$
t \triangleright_{\beta}^{1} s \Longrightarrow \Sigma(t) \triangleright_{\beta_{\mathrm{n}}} \Sigma(s)
$$

holds for all $\lambda_{p} \sigma$ terms $t$ and $s$. Then the confluence of $\triangleright$ will be an immediate consequence. In order to establish the above claim, we have to define another intermediate relation, the reduction relation $\stackrel{\beta}{\longrightarrow}$.

DEFINITION 4.15

The relation $\stackrel{\beta}{\longmapsto}$ between $\lambda_{p} \sigma$ terms and $\lambda_{p} \sigma$ substitutions is given by the following clauses (1) $-(8)$ :

\section{A. Terms}

(1) $t \stackrel{\beta}{\longmapsto} t$

(2) $(\lambda x . t) s \stackrel{\beta}{\longrightarrow} t\{s / x\}$

(3) $(\lambda x . t) \theta s \stackrel{\beta}{\longmapsto} t(s / x \cdot \theta)$

(4) $\theta \stackrel{\beta}{\longmapsto} \theta^{\prime} \Longrightarrow r \theta \stackrel{\beta}{\longmapsto} r \theta^{\prime}$

(5) $t \stackrel{\beta}{\longmapsto} s \Longrightarrow t \theta \stackrel{\beta}{\longmapsto} s \theta$

(6) $t \stackrel{\beta}{\longmapsto} s \Longrightarrow r t \stackrel{\beta}{\longmapsto} r s$

(7) $t \stackrel{\beta}{\longmapsto} s \Longrightarrow \operatorname{tr} \stackrel{\beta}{\longmapsto} s r$

\section{B. Substitutions}

(8) $t_{1} \stackrel{\beta}{\longmapsto} s_{1}, \ldots, t_{n} \stackrel{\beta}{\longmapsto} s_{n} \Longrightarrow\left\{t_{1} / x_{1}, \ldots, t_{n} / x_{n}\right\} \stackrel{\beta}{\longmapsto}\left\{s_{1} / x_{1}, \ldots, s_{n} / x_{n}\right\}$

The relation $\stackrel{\beta}{\longmapsto}$ can be considered as an extended form of one step $\beta$ reduction, where substitutions can be reduced in parallel. 
LEMMA 4.16

We have for all terms $t$ and $s$ :

$$
t \stackrel{\beta}{\longmapsto} s \Longrightarrow \Sigma(t) \triangleright_{\beta_{\mathbf{n}}} \Sigma(s)
$$

PROOF. We prove the claim by main induction on $\Psi(t)$ and side induction on the complexity of $t$. According to the structure of $t$, we can distinguish the following four cases:

(1) $t$ is a variable. Then the claim is trivial.

(2) $t$ is the term $\left(\lambda x \cdot t^{\prime}\right)$. The claim is trivial, too.

(3) $t$ is the term $\left(t_{1} t_{2}\right)$. According to $t \stackrel{\beta}{\longmapsto} s$ we can distinguish the following four subcases:

(3.1) $t^{\stackrel{\beta}{\longrightarrow}} s$ is $t_{1} t_{2} \stackrel{\beta}{\longmapsto} t_{1}^{\prime} t_{2}$ and is a consequence of $t_{1} \stackrel{\beta}{\longmapsto} t_{1}^{\prime}$. By the side induction hypothesis we have $\Sigma\left(t_{1}\right) \triangleright_{\beta_{\mathbf{n}}} \Sigma\left(t_{1}^{\prime}\right)$. This implies

$$
\Sigma\left(t_{1} t_{2}\right)=\Sigma\left(t_{1}\right) \Sigma\left(t_{2}\right) \triangleright_{\beta_{n}} \Sigma\left(t_{1}^{\prime}\right) \Sigma\left(t_{2}\right)=\Sigma\left(t_{1}^{\prime} t_{2}\right) .
$$

(3.2) $t \stackrel{\beta}{\longmapsto} s$ is $t_{1} t_{2} \stackrel{\beta}{\longmapsto} t_{1} t_{2}^{\prime}$ and is a consequence of $t_{2} \stackrel{\beta}{\longmapsto} t_{2}^{\prime}$. This case is treated in the same way as (3.1).

(3.3) $t \stackrel{\beta}{\longmapsto} s$ is $(\lambda x . r) r^{\prime} \stackrel{\beta}{\longmapsto} r\left\{r^{\prime} / x\right\}$. Then we have

$$
\Sigma\left((\lambda x . r) r^{\prime}\right)=\Sigma(\lambda x . r) \Sigma\left(r^{\prime}\right)=(\lambda x . r) \Sigma\left(r^{\prime}\right) \triangleright_{\beta_{n}} \Sigma\left(r\left\{\Sigma\left(r^{\prime}\right) / x\right\}\right)=\Sigma\left(r\left\{r^{\prime} / x\right\}\right) .
$$

(3.4) $t \stackrel{\beta}{\longmapsto} s$ is $(\lambda x . r) \theta r^{\prime} \stackrel{\beta}{\longmapsto} r\left(r^{\prime} / x \cdot \theta\right)$. First assume that $f v a r(\lambda x . r) \cap \operatorname{dom} \theta \neq 0$. Then we have for $\theta^{\prime}:=\theta\lceil(\lambda x . r)$

$$
\Sigma\left((\lambda x . r) \theta r^{\prime}\right)=(\lambda x . r) \Sigma\left(\theta^{\prime}\right) \Sigma\left(r^{\prime}\right) \triangleright_{\beta_{n}} \Sigma\left(r\left(\Sigma\left(r^{\prime}\right) / x \cdot \Sigma\left(\theta^{\prime}\right)\right)\right)=\Sigma\left(r\left(r^{\prime} / x \cdot \theta\right)\right) .
$$

The case $f v a r(\lambda x . r) \cap \operatorname{dom} \theta=\emptyset$ is treated in a similar way.

(4) $t$ is the term $\left(t^{\prime} \theta\right)$. According to the structure of $t^{\prime}$ we can distinguish the following four subcases:

(4.1) $t^{\prime}$ is the variable $x$. Then $s$ is of the form $x \theta^{\prime}$, where $\theta \stackrel{\beta}{\longmapsto} \theta^{\prime}$. If $x \notin \operatorname{dom} \theta$ then $\Sigma(x \theta)=x=\Sigma\left(x \theta^{\prime}\right)$ and there is nothing to prove. Therefore, assume $r / x \in \theta, r^{\prime} / x \in \theta^{\prime}$ and $r \stackrel{\beta}{\longmapsto} r^{\prime}$ for some terms $r$ and $r^{\prime}$. Then it is $x \theta \Delta_{\sigma}^{1} r$ and by the main induction hypothesis we have

$$
\Sigma(x \theta)=\Sigma(r) \triangleright_{\beta_{n}} \Sigma\left(r^{\prime}\right)=\Sigma\left(x \theta^{\prime}\right) .
$$

(4.2) $t^{\prime}$ is the term $\left(\lambda x \cdot t^{\prime \prime}\right)$. Then $s$ is of the form $\left(\lambda x . t^{\prime \prime}\right) \theta^{\prime}$, where $\theta \stackrel{\beta}{\longmapsto} \theta^{\prime}$. If fvar $\left(\lambda x . t^{\prime \prime}\right) \cap$ $\operatorname{dom} \theta=\emptyset$ then $\Sigma\left(\left(\lambda x . t^{\prime \prime}\right) \theta\right)=\lambda x \cdot t^{\prime \prime}=\Sigma\left(\left(\lambda x . t^{\prime \prime}\right) \theta^{\prime}\right)$ and there is nothing to prove. Otherwise, by the side induction hypothesis, we have

$$
\Sigma\left(\left(\lambda x \cdot t^{\prime \prime}\right) \theta\right)=\left(\lambda x \cdot t^{\prime \prime}\right)\left(\theta \lceil ( \lambda x \cdot t ^ { \prime \prime } ) ) \triangleright _ { \beta _ { n } } ( \lambda x \cdot t ^ { \prime \prime } ) \left(\theta^{\prime}\left\lceil\left(\lambda x \cdot t^{\prime \prime}\right)\right)=\Sigma\left(\left(\lambda x \cdot t^{\prime \prime}\right) \theta^{\prime}\right) .\right.\right.
$$

(4.3) $t^{\prime}$ is the term $\left(t_{1}^{\prime} t_{2}^{\prime}\right)$. According to $t^{\prime} \theta \stackrel{\beta}{\longmapsto} s$, we can distinguish the following five subcases: 
(4.3.1) $t^{\prime} \theta \stackrel{\beta}{\longmapsto} s$ is $\left(t_{1}^{\prime} t_{2}^{\prime}\right) \theta \stackrel{\beta}{\longmapsto}\left(t_{1}^{\prime \prime} t_{2}^{\prime}\right) \theta$ and is a consequence of $t_{1}^{\prime} \stackrel{\beta}{\longmapsto} t_{1}^{\prime \prime}$. Then it is $\left(t_{1}^{\prime} t_{2}^{\prime}\right) \theta \triangleright_{\sigma}^{1}\left(t_{1}^{\prime} \theta\right)\left(t_{2}^{\prime} \theta\right)$ and by the main induction hypothesis we have

$$
\Sigma\left(\left(t_{1}^{\prime} t_{2}^{\prime}\right) \theta\right)=\Sigma\left(\left(t_{1}^{\prime} \theta\right)\left(t_{2}^{\prime} \theta\right)\right) \triangleright_{\beta_{\mathrm{n}}} \cdot \Sigma\left(\left(t_{1}^{\prime \prime} \theta\right)\left(t_{2}^{\prime} \theta\right)\right)=\Sigma\left(\left(t_{1}^{\prime \prime} t_{2}^{\prime}\right) \theta\right) .
$$

(4.3.2) $t^{\prime} \theta \stackrel{\beta}{\longmapsto} s$ is $\left(t_{1}^{\prime} t_{2}^{\prime}\right) \theta \stackrel{\beta}{\longmapsto}\left(t_{1}^{\prime} t_{2}^{\prime \prime}\right) \theta$ and is a consequence of $t_{2}^{\prime} \stackrel{\beta}{\longmapsto} t_{2}^{\prime \prime}$. This case is treated in the same way as (4.3.1).

(4.3.3) $t^{\prime} \theta \stackrel{\beta}{\longmapsto} s$ is $\left(t_{1}^{\prime} t_{2}^{\prime}\right) \theta \stackrel{\beta}{\longmapsto}\left(t_{1}^{\prime} t_{2}^{\prime}\right) \theta^{\prime}$ and is a consequence of $\theta \stackrel{\beta}{\longmapsto} \theta^{\prime}$. Then it is $\left(t_{1}^{\prime} t_{2}^{\prime}\right) \theta \triangleright_{\sigma}^{1}\left(t_{1}^{\prime} \theta\right)\left(t_{2}^{\prime} \theta\right)$ and by the main induction hypothesis we have $\Sigma\left(t_{1}^{\prime} \theta\right) \triangleright_{\beta_{n}} \Sigma\left(t_{1}^{\prime} \theta^{\prime}\right)$ and

$\Sigma\left(t_{2}^{\prime} \theta\right) \triangleright_{\beta_{n}} \Sigma\left(t_{2}^{\prime} \theta^{\prime}\right)$. This implies

$$
\Sigma\left(\left(t_{1}^{\prime} t_{2}^{\prime}\right) \theta\right)=\Sigma\left(\left(t_{1}^{\prime} \theta\right)\left(t_{2}^{\prime} \theta\right)\right)=\Sigma\left(t_{1}^{\prime} \theta\right) \Sigma\left(t_{2}^{\prime} \theta\right) \triangleright_{\beta_{\mathrm{n}}} \Sigma\left(t_{1}^{\prime} \theta^{\prime}\right) \Sigma\left(t_{2}^{\prime} \theta^{\prime}\right)=\Sigma\left(\left(t_{1}^{\prime} t_{2}^{\prime}\right) \theta^{\prime}\right) .
$$

(4.3.4) $t^{\prime} \theta \stackrel{\beta}{\longmapsto} s$ is $\left((\lambda x . r) r^{\prime}\right) \theta \stackrel{\beta}{\longmapsto} r\left\{r^{\prime} / x\right\} \theta$. First assume that $f v a r(\lambda x . r) \cap \operatorname{dom} \theta \neq 0$. Then we have for $\theta^{\prime}:=\theta \Gamma(\lambda x . r)$

$$
\Sigma\left(\left((\lambda x . r) r^{\prime}\right) \theta\right)=(\lambda x . r) \Sigma\left(\theta^{\prime}\right) \Sigma\left(r^{\prime} \theta\right) \triangleright_{\beta_{n}} \Sigma\left(r\left(\Sigma\left(r^{\prime} \theta\right) / x \cdot \Sigma\left(\theta^{\prime}\right)\right)\right)=\Sigma\left(r\left\{r^{\prime} / x\right\} \theta\right) .
$$

The case $f \operatorname{var}(\lambda x . r) \cap \operatorname{dom} \theta=\emptyset$ is treated in a similar way.

(4.3.5) $t^{\prime} \theta \stackrel{\beta}{\longmapsto} s$ is $\left((\lambda x . r) \sigma r^{\prime}\right) \theta \stackrel{\beta}{\longmapsto} r\left(r^{\prime} / x \cdot \sigma\right) \theta$. Let us first assume that we have fvar $(\lambda x . r) \cap$ $\operatorname{dom}(\sigma \theta) \neq \theta$. Then we obtain for $\rho:=(\sigma \theta)\lceil(\lambda x . r)$

$$
\Sigma\left(\left((\lambda x . r) \sigma r^{\prime}\right) \theta\right)=(\lambda x . r) \Sigma(\rho) \Sigma\left(r^{\prime} \theta\right) \triangleright_{\beta_{n}} \Sigma\left(r\left(\Sigma\left(r^{\prime} \theta\right) / x \cdot \Sigma(\rho)\right)\right)=\Sigma\left(r\left(r^{\prime} / x \cdot \sigma\right) \theta\right) .
$$

The case $\operatorname{fvar}(\lambda x . r) \cap \operatorname{dom}(\sigma \theta)=\theta$ is treated in a similar way.

(4.4) $t^{\prime}$ is the term $\left(t^{\prime \prime} \sigma\right)$. Since $t^{\prime \prime} \sigma \theta \triangleright_{\sigma}^{1} t^{\prime \prime}(\sigma \theta)$ we can apply the main induction hypothesis to $t^{\prime \prime}(\sigma \theta)$. According to $t^{\prime} \theta \stackrel{\beta}{\longmapsto} s$ we can distinguish the following three subcases:

(4.4.1) $t^{\prime} \theta \stackrel{\beta}{\longmapsto} s$ is $t^{\prime \prime} \sigma \theta \stackrel{\beta}{\longmapsto} t^{\prime \prime} \sigma \theta^{\prime}$ and is a consequence of $\theta \stackrel{\beta}{\longmapsto} \theta^{\prime}$. Then, by the main induction hypothesis, we have

$$
\Sigma\left(t^{\prime \prime} \sigma \theta\right)=\Sigma\left(t^{\prime \prime}(\sigma \theta)\right) \triangleright_{\beta_{n}} \Sigma\left(t^{\prime \prime}\left(\sigma \theta^{\prime}\right)\right)=\Sigma\left(t^{\prime \prime} \sigma \theta^{\prime}\right) .
$$

(4.4.2) $t^{\prime} \theta \stackrel{\beta}{\longmapsto} s$ is $t^{\prime \prime} \sigma \theta \stackrel{\beta}{\longmapsto} t^{\prime \prime} \sigma^{\prime} \theta$ and is a consequence of $\sigma \stackrel{\beta}{\longmapsto} \sigma^{\prime}$. This case is treated in a very similar way to $(4.4 .1)$.

(4.4.3) $t^{\prime} \theta \stackrel{\beta}{\longmapsto} s$ is $t^{\prime \prime} \sigma \theta \stackrel{\beta}{\longmapsto} t^{\prime \prime \prime} \sigma \theta$ and is a consequence of $t^{\prime \prime} \stackrel{\beta}{\longmapsto} t^{\prime \prime \prime}$. Then, by the main induction hypothesis, we have

$$
\Sigma\left(t^{\prime \prime} \sigma \theta\right)=\Sigma\left(t^{\prime \prime}(\sigma \theta)\right) \triangleright_{\beta_{\mathrm{n}}} \Sigma\left(t^{\prime \prime \prime}(\sigma \theta)\right)=\Sigma\left(t^{\prime \prime \prime} \sigma \theta\right) .
$$

This finishes the proof of our claim.

Since $t \triangleright_{\beta}^{1} s$ trivially implies $t \stackrel{\beta}{\longmapsto} s$, we obtain the following corollary.

COROLlary 4.17

We have for all terms $t$ and $s$ :

$$
t \triangleright_{\beta}^{1} s \Rightarrow \Sigma(t) \triangleright_{\beta_{n}} \Sigma(s)
$$


Corollary 4.18

We have for all terms $t$ and $s$ :

$$
t \triangleright s \quad \Longrightarrow \quad \Sigma(t) \triangleright_{\beta_{\mathrm{n}}} \Sigma(s)
$$

Proof. Assume $t \triangleright s$. Then there is a sequence of terms $t_{1}, \ldots, t_{n}$ so that we have $t_{1}=$ $t, t_{n}=s$ and for all $1 \leq i<n$

$$
t_{i} \triangleright_{\sigma}^{1} t_{i+1} \quad \text { or } \quad t_{i} \triangleright_{\beta}^{1} t_{i+1}
$$

In the first case we have $\Sigma\left(t_{i}\right)=\Sigma\left(t_{i+1}\right)$ and in the latter, by the previous corollary, $\Sigma\left(t_{i}\right) \triangleright_{\beta_{n}} \Sigma\left(t_{i+1}\right)$. Together we immediately get $\Sigma(t) \triangleright_{\beta_{n}} \Sigma(s)$, since $\triangleright_{\beta_{n}}$ is transitively closed.

We are ready to prove the confluence of $D$.

THEOREM 4.19

$\triangle$ is Church Rosser.

PROOF. Let $t, t_{1}$ and $t_{2}$ be $\lambda_{p} \sigma$ terms and assume that

$$
t \triangleright t_{1} \quad \text { and } \quad t \triangleright t_{2}
$$

Then the previous corollary immediately implies

$$
\Sigma(t) \triangleright_{\beta_{n}} \Sigma\left(t_{1}\right) \quad \text { and } \quad \Sigma(t) \triangleright_{\beta_{n}} \Sigma\left(t_{2}\right)
$$

By Theorem 4.14 we know that $\nabla_{\beta_{n}}$ is confluent, hence there is a $\lambda_{p} \sigma$ term $s$ in substitution normal form satisfying

$$
\Sigma\left(t_{1}\right) \triangleright_{\beta_{n}} s \text { and } \Sigma\left(t_{2}\right) \triangleright_{\beta_{n}} s .
$$

Since $\nabla_{\beta_{n}} \subset \triangleright$, we obtain from (3)

$$
\Sigma\left(t_{1}\right) \triangleright s \text { and } \Sigma\left(t_{2}\right) \triangleright s
$$

which immediately implies

$$
t_{1} \triangleright \Sigma\left(t_{1}\right) \triangleright s \quad \text { and } \quad t_{2} \triangleright \Sigma\left(t_{2}\right) \triangleright s .
$$

The claim is established.

\section{Conclusion}

We have addressed some defects of the partial $\lambda$ calculus $\lambda_{p}$ as a constructive framework for partial functions. The drawbacks of $\lambda_{p}$ become even more perspicuous in the light of Pezzoli's theorem (cf. Theorem 2.9). We have proposed a modification $\lambda_{p} \sigma$ of $\lambda_{p}$ by explicit substitutions. The system $\lambda_{p} \sigma$ is embeddable into partial combinatory logic $\mathrm{CL}_{p}$, and therefore, inherits all its models. In particular, $\lambda_{p} \sigma$ has a standard interpretation in terms of ordinary recursion theory. We have studied a reduction relation for $\lambda_{p} \sigma$ and we have established a confluence result. The reduction relation gives rise to direct constructions of term models for $\lambda_{p} \sigma$. The detailed constructions will be discussed later. 


\section{Partial Applicative Theories and Explicit Substitutions}

As already mentioned, the theory of explicit substitutions has been treated before, primarily in connection with the implementation of functional programming languages. The main reference on weak calculi of explicit substitutions is [5]. In contrast to our approach, not only equality between terms, but also equality between substitutions has been axiomatized in most of the previous work on explicit substitutions (an exception is [20]). Although this can easily be achieved, the systems have much more axioms, and we think that-especially from a foundational point of view - the real concern is to axiomatize and control the notion of a substitution applied to a term, whereas equality between substitutions can be treated in the metalanguage. Furthermore, the previous systems of explicit substitutions are mainly term rewriting systems, and-this is the main difference to our $\lambda_{p} \sigma$ calculus-application in those systems is always total. The very purpose of our work, however, was to study questions of substitution in the context of partiality, and to design a more perspicuous version of the partial $\lambda$ calculus, which has natural partial models and is equivalent to partial combinatory logic.

The question arises, why should one use a fairly complicated system like $\lambda_{p} \sigma$ at all, instead of the formally more simple partial combinatory logic $\mathrm{CL}_{p}$. We think that $\lambda_{p} \sigma$ has advantages over $\mathrm{CL}_{p}$. The main reason is that in the system $\mathrm{CL}_{p}$, the intuitive clarity of the $\lambda$ notation is completely lost. Additionally, many mistakes in the literature concerning substitution in $\mathrm{CL}_{p}$ suggest that it is also worth having an explicit treatment of substitution as in $\lambda_{p} \sigma$. We, therefore, think that $\lambda_{p} \sigma$ can serve as an adequate applicative basis for systems of explicit mathematics.

As already mentioned in the introduction, Stärk (manuscript in preparation) has recently given a very natural relationship between the programming language SCHEME and the $\lambda_{p} \sigma$ calculus. In his approach, partiality of $\lambda_{p} \sigma$ is essential. The results of Stärk give further evidence for the foundational significance of the $\lambda_{p} \sigma$ calculus.

\section{Acknowledgments}

I am indebted to R. Kahle, who pointed out to me that the principle $(\star \star)$ is derivable in $\lambda_{p}$ though it obviously does not hold in the recursion-theoretic model, and to E. Pezzoli for providing the crucial Theorem 2.9. I also thank A. S. Troelstra for calling my attention to explicit substitutions as well as S. Feferman, T. Hardin, G. Jäger, R. Stärk, and W. Th. Wolff for helpful comments on an earlier version of this paper.

Research supported by the Swiss National Science Foundation.

\section{References}

[1] M. Abadi, L. Cardelli, P.-L. Curien, and J.-J. Lévy. Explicit substitutions. Joumal of Functional Programming, 1, 375-416, 1991.

[2] M. J. Becson. Foundations of Constructive Mathematics: Metamathematical Studies. Springer, Berlin, 1984.

[3] M. J. Beeson. Proving programs and programming proofs. In Logic, Methodology and Philosophy of Science VII, R. Barcan Marcus, G. J. W. Dorn and P. Weingartner, eds, pp. 51-82. North Holland, Amsterdam, 1986.

[4] P.-L. Curien. An abstract framework for environment machines. Theoretical Computer Science, 82, 389-402, 1991.

[5] P.-L. Curien, T. Hardin and J.-J. Levy. Confluence properties of weak and strong calculi of explicit substitutions. Technical Report 92-01, CEDRIC, 292 rue Saint-Martin, 75141 Paris Cédex 03, France, 1992. Submitted to J.A.C.M.

[6] S. Feferman. A language and axioms for explicit mathematics. In Algebra and Logic, Vol. 450 of Lecture Notes in Mathematics, J. N. Crosstey, ed., pp. 87-139. Springer, Berlin, 1975. 
[7] S. Feferman. Constructive theories of functions and classes. In Logic Colloquium '78, M. Boffa, D. van Dalen and K. McAloon, eds, pp. 159-224. North Holland, Amsterdam, 1979.

[8] S. Feferman. Polymorphic typed lambda-calculi in a type-free axiomatic framework. In Logic and Computation, Vol. 106 of Contemporary Mathematics, W. Sieg, ed., pp. 101-136. American Mathematical Society, Providence, Rhode Island, 1990.

[9] S. Feferman. Logics for termination and correctness of functional programs. In Logic from Computer Science, Vol. 21 of MSRI Publications, Y. N. Moschovakis, ed., pp. 95-127. Springer, Berlin, 1991.

[10] S. Feferman. Logics for termination and correctness of functional programs II: Logics of strength PRA. In Proof Theory, P. Aczel, H. Simmons and S. S. Wainer, eds, pp. 195-225. Cambridge University Press, Cambridge. 1992.

[11] S. Feferman and G. Jăger. Systems of explicit mathematics with non-constructive $\mu$-operator. Part l. Annals of Pure and Applied Logic, 65, 243-263, 1993.

[12] T. Hardin. Confluence results for the pure strong categorical logic CCL: $\lambda$-calculi as subsystems of CCL. Theoretical Computer Science, 65, 291-342, 1989.

[13] T. Hardin and J.-J. Lévy. A confluent calculus of substitutions. Technical Report 90-11, CEDRIC, 292 rue Saint-Martin, 75141 Paris Cedex 03, France, 1990.

[14] J. R. Hindley. Combinatory reductions and lambda reductions compared. Zeitschrift fur mathematische Logik und Grundlagen der Mathemarik, 23, 169-180, 1977.

[15] J. R. Hindley and G. Longo. Lambda-calculus models and extensionality. Zeitschrift fur mathematische Logik und Grundlagen der Mathematik, 26, 289-310, 1980.

[16] G. Jugger. Induction in the elementary theory of types and names. In Computer Science Logic '87, Vol. 329 of Lecture Notes in Computer Science, E. Börger, H. Kleine Buining and M. M. Richter, ods, pp. 118-128. Springer. Berlin, 1988.

[17] G. Jłger. Type theory and explicit mathematics. In Logic Colloquium '87, H.-D. Ebbinghaus, J. FernandezPrida, M. Garrido, M. Lascar and M. Rodriguez Artalejo, eds, pp. 117-135. North Holland, Amsterdam, 1989.

[18] G. Jiger and Th. Strahm. Totality in applicative theories. Annals of Pure and Applied Logic. Accepted for publication.

[19] S. C. Kleene. Introduction to Metamathematics. North Holland, Amsterdam, 1952.

[20] P. Lescanne and J. Rouyer-Degli. The calculus of explicit substitution $\lambda v$. Technical report, CNRS and INRIALorraine, Campus Scientifique, BP 239, 54506 Vandœuvre-les-Nancy, France, March 1994.

[21] P. Martin-Lof. Substitition calculus. Unpublished notes, September 1992.

[22] M. Marzetta. A theory of types and names. Contributed paper, Logic Colloquium, Padova 23-30 August 1988.

[23] M. Marzetta. Universes in the theory of types and names. In Computer Science Logic '92, Vol. 702 of Lecture Notes in Computer Science, E. Börger, G. Jäger, H. Kleine Buning, S. Martin and M. M. Richter, eds, pp. 340 351. Springer, Berlin, 1993.

[24] E. Moggi. The partial lambda calculus. PhD thesis, University of Edinburgh, 1988.

[25] M. H. A. Newman. On theories with a combinatorial definition of "equivalence". Annals of Mathematics, 43, 223-243, 1942.

[26] A. Tasistro. Formulation of Martin-Lof's theory of types with explicit substitutions. Technical report, Department of Computer Sciences, University of Göteborg, May 1993.

[27] A. S. Troelstra and D. van Dalen. Constructivism in Mathematics, Vol. I. North Holland, Amsterdam, 1988.

[28] A. S. Troelstra and D. van Dalen. Constructivism in Mathematics, Vol. II. North Holland, Amsterdam, 1988.

Received 29 June 1993 